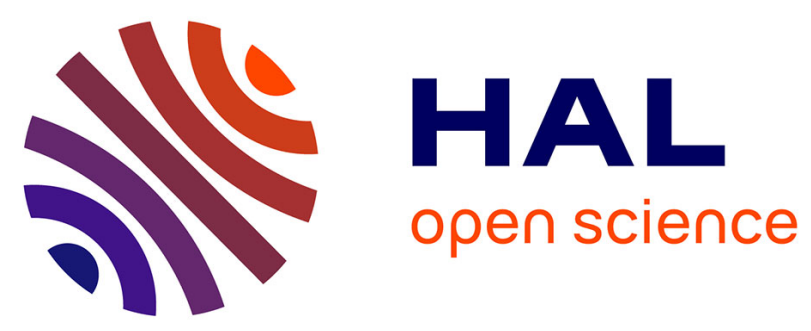

\title{
An analytical framework for the numerical homogenization of monotone elliptic operators and quasiconvex energies
}

Antoine Gloria

\section{- To cite this version:}

Antoine Gloria. An analytical framework for the numerical homogenization of monotone elliptic operators and quasiconvex energies. Multiscale Modeling and Simulation: A SIAM Interdisciplinary Journal, 2006, 5 (3), pp.996-1043. 10.1137/060649112 . inria-00070230

\section{HAL Id: inria-00070230 \\ https://hal.inria.fr/inria-00070230}

Submitted on 19 May 2006

HAL is a multi-disciplinary open access archive for the deposit and dissemination of scientific research documents, whether they are published or not. The documents may come from teaching and research institutions in France or abroad, or from public or private research centers.
L'archive ouverte pluridisciplinaire HAL, est destinée au dépôt et à la diffusion de documents scientifiques de niveau recherche, publiés ou non, émanant des établissements d'enseignement et de recherche français ou étrangers, des laboratoires publics ou privés. 
INSTITUT NATIONAL DE RECHERCHE EN INFORMATIQUE ET EN AUTOMATIQUE

\section{An analytical framework for the numerical homogenization of monotone elliptic operators and quasiconvex energies}

Antoine Gloria

\section{$\mathbf{N}^{\circ} 5791$ - version 2}

version initiale Décembre, 2005 - version révisée Janvier, 2006

Thème NUM 



\title{
An analytical framework for the numerical homogenization of monotone elliptic operators and quasiconvex energies
}

\author{
Antoine Gloria* \\ Thème NUM — Systèmes numériques \\ Projet MICMAC \\ Rapport de recherche $\mathrm{n}^{\circ} 5791$ - version $2^{\dagger}$ - version initiale Décembre, 2005 - version \\ révisée Janvier, 200644 pages
}

\begin{abstract}
A number of methods have been proposed in the recent years to perform the numerical homogenization of (possibly nonlinear) elliptic operators. These methods are usually defined at the discrete level. Most of them compute a numerical operator, close, in a sense to be made precise, to the homogenized elliptic operator for the problem. The purpose of the present work is to clarify the construction of this operator in the convex case by interpreting the method at the continuous level and to extend it to the nonconvex setting. The discretization of this new operator may be performed in several ways, recovering and generalizing a variety of methods, such as the multiscale finite element method (MsFEM) or the heterogeneous multiscale method (HMM). In addition to the above, we introduce an original and general numerical corrector in the convex case.
\end{abstract}

Key-words: homogenization, $\Gamma$-convergence, elliptic operator, quasiconvexity, HMM, MsFEM, corrector, coarse graining

* CERMICS, Ecole Nationale des Ponts et Chaussées, 6 et 8 avenue Blaise Pascal, Cité Descartes, 77455 Marne-la-Vallée Cedex 2, France, and MICMAC, INRIA, Rocquencourt. Contact: antoine.gloria@inria.fr

$\dagger$ This revised report corrects some misstatements of the first version and extends the numerical analysis 


\section{Analyse et homogénéisation numérique des opérateurs elliptiques monotones et des énergies quasiconvexes}

Résumé : De nombreuses méthodes ont été proposées ces dernières années pour homogénéiser numériquement des opérateurs elliptiques linéaires ou non linéaires. Ces méthodes sont souvent définies au niveau discret. La plupart d'entre elles consiste à calculer numériquement un nouvel opérateur, qui approxime, en un sens à préciser, l'opérateur homogénéisé du problème. L'objectif de ce travail est de clarifier la construction de cet opérateur numérique dans le cas convexe en introduisant une version continue de l'opérateur, de généraliser l'approche au cas quasiconvexe et d'en faire l'analyse. Ce nouvel opérateur peut alors être discrétisé de différentes façons, retrouvant ainsi différentes méthodes comme les éléments finis multiéchelles ou la méthode HMM. En outre, nous introduisons un correcteur numérique dans le cas convexe général.

Mots-clés : homogénéisation, $\Gamma$-convergence, opérateur elliptique, quasiconvexité, HMM, $\mathrm{MsFEM}$, correcteur, coarse graining 


\section{Setting of the problem and statement of the main re- sults}

For the sake of simplicity and in order to relate the present work to existing approaches, we first consider a scalar elliptic partial differential equation that is the Euler-Lagrange equation of a problem of minimization of an energy. The energy density is assumed to be convex and to vary at a scale small with respect to the size of the domain, which makes direct numerical simulations impossible to perform in practice. Our purpose is to introduce an averaged energy density which does not vary as much as the original energy density and prove that the associated minimization problem is a correct approximation of the original minimization problem in the sense of $\Gamma$-convergence.

Although strict convexity ensures the existence and uniqueness of the solution to the Euler-Lagrange equation and allows to use a more direct approach (e.g. $G$-convergence), our arguments are based on variational principles. Most of the existing works (see e.g. [9], [10], [8]) actually treat the PDE. Our approach allows to deal with the case of nonlinear elasticity, as will be seen. The first Section is dedicated to the introduction of the averaged energy density in the framework of the homogenization theory for convex energies. Theorem 1 states the $\Gamma$-convergence of the averaged energy to the homogenized energy and Theorem 2 introduces a new general corrector result, which describes the fine scales features of the solution of the original minimization problem without assumption on the heterogeneities. This corrector is called a numerical corrector in reference to [10], where it was first derived in the stochastic and stationary case. Section 2 is dedicated to the proofs of the main theorems.

Besides the analysis of convex problems, a generalization of this approach is introduced in Section 1.3 to deal with nonconvex energy densities. Definition 10 and Theorem 3 are the natural counterparts of Definition 3 and Theorem 1 for quasiconvex energy densities. The proof of Theorem 1 is adapted to the quasiconvex case in Section 2.3.

Finally, Section 3 relates the present work to some well-known numerical methods such as the MsFEM and the HMM to which the present analysis applies both in the convex and quasiconvex settings.

We assume the reader is familiar with the basic properties of the $\Gamma$-convergence theory. Should the need arise, [5] provides with a good introduction and [6] gives a more systematic study of the subject. For consistency, let us recall some notation and properties of the $\Gamma$-convergence in Sobolev spaces. In the sequel, $\Omega$ denotes an open bounded subset of $\mathbb{R}^{n}$ $(n \geq 1), W^{1, p}(\Omega)$ denotes the Sobolev space for $p \geq 1$ and $p^{\prime}$ denotes the conjugate exponent defined by $\frac{1}{p}+\frac{1}{p^{\prime}}=1$.

Definition 1 Let $F_{\epsilon}: W^{1, p}\left(\mathbb{R}^{n}\right) \rightarrow \mathbb{R}$ be a family of functions. We say that $F_{\epsilon} \Gamma\left(L^{p}\right)$ converges (resp. $\Gamma\left(W^{1, p}\right)$-converges) to $F: W^{1, p}\left(\mathbb{R}^{n}\right) \rightarrow \mathbb{R}$ on $\Omega$ if and only if the two following properties are satisfied.

$\mathrm{RR} \mathrm{n}^{\circ} 5791$ 
(i) Liminf inequality: for every $u \in W^{1, p}(\Omega)$ and every sequence $u_{\epsilon}$ such that $u_{\epsilon} \rightarrow u$ (resp. $\left.u_{\epsilon} \rightarrow u\right)$ in $W^{1, p}(\Omega)$,

$$
F(u) \leq \liminf _{\epsilon} F_{\epsilon}\left(u_{\epsilon}\right) .
$$

(ii) Recovery sequence: for every $u \in W^{1, p}(\Omega)$ there exists a sequence $\bar{u}_{\epsilon}$ such that $\bar{u}_{\epsilon} \rightarrow u$ $\left(\right.$ resp. $\left.\bar{u}_{\epsilon} \rightarrow u\right)$ in $W^{1, p}(\Omega)$ and

$$
\limsup _{\epsilon} F_{\epsilon}\left(\bar{u}_{\epsilon}\right) \leq F(u) .
$$

Definition 1 is also refered to as the sequential $\Gamma$-convergence since it is stated using the convergence of sequences. We refer to [5, Section 1.4] for equivalent definitions.

The $\Gamma$-convergence implies the convergence of minima and minimizers of functions as stated in the following

Lemma 1 Let $F_{\epsilon}: W^{1, p}\left(\mathbb{R}^{n}\right) \rightarrow \mathbb{R}$ be a family of functions that $\Gamma\left(L^{p}\right)$-converges to $F$ on $\Omega$. If $F_{\epsilon}$ is lower semicontinuous for the weak topology of $W^{1, p}\left(\mathbb{R}^{n}\right)$ and equicoercive in the following sense

$$
\exists \quad c>0, \quad \forall v \in W^{1, p}(\Omega), \forall \epsilon>0, \quad c\|\nabla v\|_{L^{p}(\Omega)}^{p} \leq F_{\epsilon}(v)
$$

then for every $u_{0} \in W^{1, p}(\Omega)$

$$
\lim _{\epsilon \rightarrow 0}\left(\inf \left\{F_{\epsilon}\left(v+u_{0}\right), v \in W_{0}^{1, p}(\Omega)\right\}\right)=\inf \left\{F\left(v+u_{0}\right), v \in W_{0}^{1, p}(\Omega)\right\}
$$

and for every sequence $u_{\epsilon}$ of minimizers of $\inf \left\{F_{\epsilon}\left(v+u_{0}\right), v \in W_{0}^{1, p}(\Omega)\right\}$ there exists a subsequence (not relabeled) and a minimizer $u$ of $\inf \left\{F\left(v+u_{0}\right), v \in W_{0}^{1, p}(\Omega)\right\}$ such that $u_{\epsilon} \rightarrow u$ in $W^{1, p}(\Omega)$.

In the following, $\Gamma$ denotes the $\Gamma\left(L^{p}\right)$-convergence. For all open bounded subset $\mathcal{O}$ of $\mathbb{R}^{n}$ and $u \in L^{1}(\mathcal{O})$, we denote

$$
<u>_{\mathcal{O}}=\frac{1}{\mathcal{L}^{n}(\mathcal{O})} \int_{\mathcal{O}} u(x) d x
$$

\subsection{Homogenization of convex energy densities}

Let us first recall classical homogenization results for convex energy densities (see [4], [6] and $[18])$.

Definition 2 A function $W: \mathbb{R}^{n} \times \mathbb{R}^{n} \rightarrow \mathbb{R}$ is a Carathéodory function if for every $\xi \in \mathbb{R}^{n}$, $W(\cdot, \xi)$ is measurable and if for almost all $x \in \mathbb{R}^{n}, W(x, \cdot)$ is continuous.

A Carathéodory function on $\mathbb{R}^{n} \times \mathbb{R}^{n}$ is a Borel function on $\mathbb{R}^{n} \times \mathbb{R}^{n}$.

Lemma 2 Let $W_{\epsilon}: \mathbb{R}^{n} \times \mathbb{R}^{n} \rightarrow[0,+\infty)$ be a set of functions satisfying the following conditions:

INRIA 
- H1: $W_{\epsilon}$ is a Carathéodory function;

- H2: for almost every $x \in \mathbb{R}^{n}, W_{\epsilon}(x, \cdot)$ is convex;

- H3: there exist $0<c \leq C$ and $p \geq 1$ such that

$$
c|\xi|^{p} \leq W_{\epsilon}(x, \xi) \leq C\left(1+|\xi|^{p}\right)
$$

for almost all $x \in \mathbb{R}^{n}$ and for all $\xi \in \mathbb{R}^{n}$.

Consider $\Omega$ a bounded open subset of $\mathbb{R}^{n}$ and set for all $\epsilon>0$,

$$
F_{\epsilon}(u)=\int_{\Omega} W_{\epsilon}(x, \nabla u(x)) d x
$$

for all $u \in W^{1, p}(\Omega, \mathbb{R})$. Then, up to extraction, there exists a function $W_{\text {hom }}$ satisfying $H 1$, H2 and H3, such that we have

$$
\Gamma\left(L^{p}\right)-\lim _{\epsilon \rightarrow 0} F_{\epsilon}(u)=\int_{\Omega} W_{h o m}(x, \nabla u(x)) d x,
$$

for all $u \in W^{1, p}(\Omega, \mathbb{R})$.

Lemma 3 In addition to H1, H2 and H3, let assume that $p \geq 2$, that

- $H_{4}: W_{\epsilon}(x, \cdot)$ is continuously differentiable for almost all $x \in \Omega$ and $a_{\epsilon}(\cdot, 0)=\frac{\partial W_{\epsilon}}{\partial \xi}(\cdot, 0)$ is bounded;

and that the following monotonicity and continuity properties hold:

$$
\begin{gathered}
\exists 0 \leq \alpha \leq p-1, C>0 \quad \mid \quad \text { for almost all } x \in \mathbb{R}^{n}, \text { for all } \xi_{1}, \xi_{2} \in \mathbb{R}^{n} \\
\qquad\left|a_{\epsilon}\left(x, \xi_{1}\right)-a_{\epsilon}\left(x, \xi_{2}\right)\right| \leq C\left(1+\left|\xi_{1}\right|+\left|\xi_{2}\right|\right)^{p-1-\alpha}\left|\xi_{1}-\xi_{2}\right|^{\alpha}, \\
\exists 2 \leq \beta<+\infty, c>0 \quad \mid \quad \text { for almost all } x \in \mathbb{R}^{n}, \text { for all } \xi_{1}, \xi_{2} \in \mathbb{R}^{n} \\
\quad\left(a_{\epsilon}\left(x, \xi_{1}\right)-a_{\epsilon}\left(x, \xi_{2}\right), \xi_{1}-\xi_{2}\right) \geq c\left(1+\left|\xi_{1}\right|+\left|\xi_{2}\right|\right)^{p-\beta}\left|\xi_{1}-\xi_{2}\right|^{\beta} .
\end{gathered}
$$

Then, given $f \in L^{p^{\prime}}(\Omega)$, the solution $u_{\epsilon} \in W_{0}^{1, p}(\Omega)$ to

$$
-\operatorname{div}\left(a_{\epsilon}\left(x, \nabla u_{\epsilon}\right)\right)=f
$$

weakly converges in $W_{0}^{1, p}(\Omega)$, up to extraction, to the solution $u \in W_{0}^{1, p}(\Omega)$ to

$$
-\operatorname{div}\left(a_{\text {hom }}(x, \nabla u)\right)=f \text {, }
$$

where $a_{\text {hom }}: \mathbb{R}^{n} \times \mathbb{R}^{n} \rightarrow \mathbb{R}^{n}$ is related to $W_{\text {hom }}$ defined in Lemma 2 by

$$
a_{\text {hom }}=\frac{\partial W_{h o m}}{\partial \xi} .
$$

In addition $a_{\text {hom }}$ satisfies (3) with the same coefficient $c$ and exponent $\beta$, and (2) with the same coefficient $C$ and the exponent $\alpha /(\beta-\alpha)$ instead of $\alpha$.

$\mathrm{RR} \mathrm{n}^{\circ} 5791$ 


\subsection{Main results}

Let $\left(W_{\epsilon}\right)$ be a family of energy densities satisfying the assumptions $H 1, H 2$ and $H 3$. The problem we consider is

$$
\inf \left\{\int_{\Omega} W_{\epsilon}(x, \nabla u), u \in W^{1, p}(\Omega)+B C\right\} .
$$

By $W^{1, p}(\Omega)+B C$ in $(4)$, we mean any subspace of $W^{1, p}(\Omega)$ associated to usual boundary conditions. In particular, we will consider

(1) $\phi+W_{0}^{1, p}(\Omega)$ for any $\phi \in W^{1, p}(\Omega)$,

(2) $\left\{u \in W^{1, p}(\Omega) \mid u(x)=\xi \cdot x+v(x), v \in W_{\#}^{1, p}(\Omega)\right\}$ for any $\xi \in \mathbb{R}^{n}$, with $W_{\#}^{1, p}(\Omega)=$ $\left\{v_{\mid \Omega} \mid v \in W_{l o c}^{1, p}\left(\mathbb{R}^{n}\right), v\right.$ is $\Omega$-periodic $\}$ if $\mathbb{R}^{n}$ can be obtained by the periodic replication of $\Omega$,

(3) $\left\{u \in W^{1, p}(\Omega) \mid<\nabla u>_{\Omega}=\xi\right\}$ for any $\xi \in \mathbb{R}^{n}$.

These three boundary conditions indeed do not influence the energy density of the $\Gamma$ limit of (4), as briefly recalled in the Appendix. This is why we do not make them specific in the sequel.

Up to extraction, problem (4) $\Gamma$-converges to

$$
\inf \left\{\int_{\Omega} W_{h o m}(x, \nabla u), u \in W^{1, p}(\Omega)+B C\right\} .
$$

The density $W_{\text {hom }}$ is not explicitly known. Lemma 2 only provides with an existence result.

For brevity, we denote by

$$
\begin{array}{r}
I_{\epsilon}(u)=\int_{\Omega} W_{\epsilon}(x, \nabla u), \\
I_{h o m}(u)=\int_{\Omega} W_{h o m}(x, \nabla u),
\end{array}
$$

for $u \in W^{1, p}(\Omega)$.

We now introduce a notion of energy averages on balls.

Definition 3 For any $\eta>0$, denoting by $B(x, \eta)$ the ball of radius $\eta$ centered at point $x \in \mathbb{R}^{n}$, we define the energy density

$$
W_{\eta, \epsilon}(x, \xi)=\inf \left\{<W_{\epsilon}(\cdot, \nabla v(\cdot))>_{B(x, \eta)} \mid v \in W^{1, p}(B(x, \eta)),<\nabla v>_{B(x, \eta)}=\xi\right\}
$$

from $\mathbb{R}^{n} \times \mathbb{R}^{n}$ to $\mathbb{R}$ and the associated energy functional

$$
I_{\eta, \epsilon}(u)=\int_{\Omega} W_{\eta, \epsilon}(x, \nabla u), \text { for all } u \in W^{1, p}(\Omega) .
$$


Remark 1 We have used balls $B(x, \eta)$ for defining averaged energies. All the results presented throughout this work hold for generic open neighborhoods $N(x, \eta)$ satisfying that for every $x \in \Omega$, there exist $0<c \leq C$ such that for every $\eta>0, c|B(x, \eta)| \leq|N(x, \eta)| \leq$ $C|B(x, \eta)|$.

Let us introduce the concept of "equi isolated minimizers" before stating the first main result.

Definition 4 Given a family of energy functionals $F_{N}$ on the metric space $(V, d)$, we say that a family $u_{N}$ of minimizers of $F_{N}$ on $(V, d)$ is equi isolated if there exists a ball $B \subset V$ such that $u_{N} \in B$ and $u_{N}$ is the unique minimizer of $F_{N}$ on $B$ for every $N>0$.

Our first result is the following

Theorem 1 For $p>1$, the energy densities $W_{\eta, \epsilon}$ satisfy H1, H2 and H3 and the energy $I_{\eta, \epsilon} \Gamma\left(L^{p}\right)$ and $\Gamma\left(W^{1, p}\right)$-converges to $I_{h o m}$ as $\epsilon$ and $\eta$ go to 0 . Therefore, for any sequence $u_{\eta, \epsilon}$ of minimizers of $\inf \left\{I_{\eta, \epsilon}(v) \mid v \in W^{1, p}(\Omega, \mathbb{R})+B C\right\}$, there exists a minimizer $u_{\text {hom }}$ of $\inf \left\{I_{h o m}(v) \mid v \in W^{1, p}(\Omega, \mathbb{R})+B C\right\}$ such that

$$
\lim _{\eta \rightarrow 0} \lim _{\epsilon \rightarrow 0} u_{\eta, \epsilon}=u_{\text {hom }} \quad \text { weakly in } W^{1, p}(\Omega, \mathbb{R}),
$$

up to extraction.

Correspondingly, for any minimizer $u_{\text {hom }}$ of $\inf \left\{I_{h o m}(v) \mid v \in W^{1, p}(\Omega, \mathbb{R})+B C\right\}$ there exists a sequence $u_{\eta, \epsilon}$ of minimizers of $\inf \left\{I_{\eta, \epsilon}(v) \mid v \in W^{1, p}(\Omega, \mathbb{R})+B C\right\}$ such that

$$
\lim _{\eta \rightarrow 0} \lim _{\epsilon \rightarrow 0} u_{\eta, \epsilon}=u_{\text {hom }} \quad \text { strongly in } W^{1, p}(\Omega, \mathbb{R}) .
$$

In addition, if $u_{\eta, \epsilon}$ is a family of equi isolated minimizers in the sense of Definition 4 then $u_{\text {hom }}$ is also isolated and (8) holds.

In particular, if $W_{\epsilon}(x, \cdot)$ is strictly convex almost everywhere on $\mathbb{R}^{n}$, the unique sequence of minimizers $u_{\eta, \epsilon}$ strongly converges in $W^{1, p}(\Omega, \mathbb{R})$ to the unique minimizer $u_{\text {hom }}$.

Remark 2 The order of the limits in (7) is important and cannot be changed in general.

We can also define a set of numerical correctors to approximate $\nabla u_{\epsilon}$ in $L^{p}(\Omega)$.

Definition 5 Let $\left\{Q_{H, i}\right\}_{i \in \llbracket 1, I_{H} \rrbracket}$ be a partition of $\Omega$ in disjoint subdomains of diameter of order $H$. We define a family $\left(M_{H}\right)$ of approximations of identity on $L^{p}(\Omega)$ associated to $Q_{H, i}:$ for every $w \in L^{p}(\Omega)$ and $H>0$,

$$
M_{H}(w)=\sum_{i=1}^{I_{H}}<w>_{Q_{H, i}} 1_{Q_{H, i}} .
$$

$\mathrm{RR} \mathrm{n}^{\circ} 5791$ 
Keeping the notation of Theorem 1, we define the numerical correctors $v_{\eta, \epsilon}^{H, i}$ for a strictly convex energy density as the unique minimizers of

$$
\inf \left\{\int_{Q_{H, i}} W_{\epsilon}(x, \nabla v) \mid v \in W^{1, p}\left(Q_{H, i}\right),<\nabla v>_{Q_{H, i}}=M_{H}\left(\nabla u_{\eta, \epsilon}\right)_{\mid Q_{H, i}}\right\} .
$$

Theorem 2 Assume $p \geq 2, H 1, H 2, H 3, H_{4}$, (2) and (3) with $\beta \leq p$. We keep the notation of Lemma 3, Theorem 1 and Definition 5. We have

$$
\lim _{\eta, H \rightarrow 0} \lim _{\epsilon \rightarrow 0}\left\|\nabla u_{\epsilon}-\sum_{i=1}^{I_{H}} \nabla v_{\eta, \epsilon}^{H, i} 1_{Q_{H, i}}\right\|_{L^{p}(\Omega)}=0 .
$$

Remark 3 The order of the limits in $H$ and $\eta$ in (10) is not important and we may take e.g. $H=\eta \rightarrow 0$. However we have to first let $\epsilon$ go to zero.

Remark 4 Theorem 1 holds if $W_{\eta, \epsilon}(x, \xi)$ is replaced by

$$
\inf \left\{<W_{\epsilon}(\cdot, \nabla v(\cdot))>_{B(x, \eta)} \mid v \in W^{1, p}(B(x, \eta)), v(y)=\xi \cdot y \text { on } \partial B(x, \eta)\right\} .
$$

Theorem 2 also holds if $v_{\eta, \epsilon}^{H, i}$ is replaced by the unique solution in $W^{1, p}\left(Q_{H, i}\right)$ of

$$
\inf \left\{\int_{Q_{H, i}} W_{\epsilon}(x, \nabla v) \mid v(x)=M_{H}\left(\nabla u_{\eta, \epsilon}\right)_{\mid Q_{H, i}} \cdot x \text { on } \partial Q_{H, i}\right\} \text {. }
$$

The proofs of Section 2 can be easily adapted to these cases.

Remark 5 If the neighborhood $N(x, \eta)$ is such that $\mathbb{R}^{n}$ can be obtained by the periodic replication of $N(x, \eta)$ then Theorem 1 holds with

$$
W_{\eta, \epsilon}(x, \xi)=\inf \left\{<W_{\epsilon}(\cdot, \xi+\nabla v(\cdot))>_{N(x, \eta)} \mid v \in W_{\#}^{1, p}(N(x, \eta))\right\} .
$$

From a theoretical point of view, $u_{\eta, \epsilon}$ has the advantage to strongly converge to $u_{\text {hom }}$ in $W^{1, p}(\Omega)$, whereas $u_{\epsilon}$ only converges weakly. This has an important consequence on the practical numerical computation of $u_{\eta, \epsilon}$ and $u_{\epsilon}$. As $u_{\eta, \epsilon}$ strongly converges to $u_{h o m}$, the gradient of $u_{\eta, \epsilon}$ does not oscillate at order 1 at the period $\epsilon$ when $\eta$ and $\epsilon$ are sufficiently small if $\nabla u_{\text {hom }}$ does not oscillate at small scales. This may allow to take a meshsize larger than $\epsilon$ to approximate $u_{\eta, \epsilon}$ with a finite element method. This is not the case for $u_{\epsilon}$. In fact, state of the art multiscale methods for elliptic equations usually compute numerical approximations of $u_{\eta, \epsilon}$, as will be seen in Section 3 .

\subsection{Extension to the quasiconvex case}

Let us briefly recall the corresponding version of Lemma 2 for quasiconvex energy densities.

INRIA 
Definition 6 Given $p \geq 1, n \geq 1$ and $d \geq 1$, a function $W: \mathbb{R}^{n \times d} \rightarrow[0,+\infty]$ is $W^{1, p}$ quasiconvex (or simply quasiconvex in the sequel) if for all $A \in \mathbb{R}^{n \times d}$, there exists an open bounded subset $E$ of $\mathbb{R}^{n}$ with $\mathcal{L}^{n}(\partial E)=0$ such that:

$$
W(A)=\min \left\{\frac{1}{\mathcal{L}^{n}(E)} \int_{E} W(A+\nabla \phi(x)) d x \mid \phi \in W_{0}^{1, p}\left(E ; \mathbb{R}^{3}\right)\right\} .
$$

Remark 6 A quasiconvex function is rank-one convex, that is for every rank-one matrix $\xi \in \mathbb{R}^{n \times d}$ and for every $\zeta \in \mathbb{R}^{n \times d}$, the function $\mathbb{R} \ni t \mapsto W(\zeta+t \xi)$ is convex.

The characteristics of quasiconvexity is to ensure the weak-lower semicontinuity of integral functionals.

Lemma 4 If $1 \leq p<\infty$, and $W: \mathbb{R}^{n \times d} \rightarrow \mathbb{R}$ is a quasiconvex function satisfying

$$
0 \leq W(A) \leq C\left(1+|A|^{p}\right) \quad \text { for all } A \in \mathbb{R}^{n \times d},
$$

then the functional $J: u \mapsto \int_{\Omega} W(\nabla u)$ is weakly lower semi-continuous on $W^{1, p}(\Omega)$.

Definition 7 [5, Section 12.1] Given a continuous function $f: \mathbb{R}^{n \times d} \rightarrow \mathbb{R}, \xi \mapsto f(\xi)$ that satisfies (12), its quasiconvex envelop $Q f$ is defined as the greatest quasiconvex function lower or equal to $f$. In particular, it is given for all $\xi \in \mathbb{R}^{n \times d}$ by

$$
\begin{aligned}
Q f(\xi) & =\inf \left\{\int_{(0,1)^{n}} f(\xi+\nabla u(y)) d y \mid u \in W_{0}^{1, p}\left((0,1)^{n}, \mathbb{R}^{d}\right)\right\} \\
& =\inf \left\{\int_{(0,1)^{n}} f(\xi+\nabla u(y)) d y \mid u \in W_{\#}^{1, p}\left((0,1)^{n}, \mathbb{R}^{d}\right)\right\} .
\end{aligned}
$$

Definition 8 Let $(x, A) \mapsto W(x, A)$ be a Carathéodory function defined on $\mathbb{R}^{n} \times \mathbb{R}^{n \times d}$, for which there exist an integer $p \geq 1$, positive constants $c$ and $C$, such that for almost all $x \in \mathbb{R}^{n}$ and for all $A \in \mathbb{R}^{n \times d}$,

$$
c|A|^{p} \leq W(x, A) \leq C\left(1+|A|^{p}\right)
$$

The function $W$ is then said to satisfy a standard growth condition (of order $p$ ).

Definition 9 The function $W: \mathbb{R}^{n} \times \mathbb{R}^{n \times d} \rightarrow \mathbb{R},(x, A) \mapsto W(x, A)$ is a standard energy density if $W$ is a quasiconvex Carathéodory function, that is:

- $W(\cdot, \cdot)$ is measurable in its first variable and continuous in its second variable

- $W(x, \cdot)$ is quasiconvex for almost every $x \in \mathbb{R}^{n}$

and if $W$ satisfies (12).

$\mathrm{RR} \mathrm{n}^{\circ} 5791$ 
We are now in position to recall the homogenization result for quasiconvex energy densities.

Lemma 5 [4, Theorem 12.5] Let $W_{\epsilon}: \mathbb{R}^{n} \times \mathbb{R}^{n \times d} \rightarrow[0,+\infty)$ be a set of standard energy densities satisfying the growth condition (12) of order $p>1$ uniformly in $\epsilon$. For any bounded open subset $\Omega$ of $\mathbb{R}^{n}$, for all $u \in W^{1, p}\left(\Omega, \mathbb{R}^{d}\right)$ and $\epsilon>0$, we set

$$
I_{\epsilon}(u)=\int_{\Omega} W_{\epsilon}(x, \nabla u(x)) d x .
$$

Then, there exists a homogenized standard energy density $W_{\text {hom }}: \Omega \times \mathbb{R}^{n \times d} \rightarrow[0,+\infty)$ satisfying (12) and such that, up to extraction, $\Gamma\left(L^{p}\right)-\lim _{\epsilon \rightarrow 0} I_{\epsilon}=I_{\text {hom }}$ on $W^{1, p}\left(\Omega, \mathbb{R}^{d}\right)$ where $I_{h o m}(u)=\int_{\Omega} W_{h o m}(x, \nabla u(x)) d x$.

Contrary to the convex case, quasiconvexity is not preserved by the averaging of Definition 3. Therefore we have to use quasiconvex envelops to obtain results corresponding to Theorem 1.

Definition 10 For $\eta>0, C(x, \eta)$ is the hypercube of $\mathbb{R}^{n}$ centered in $x \in \mathbb{R}^{n}$ and of length $\eta$. We then define the averaged energy density by

$$
\mathcal{W}_{\eta, \epsilon}(x, \xi)=\inf \left\{<W_{\epsilon}(\cdot, \xi+\nabla v(\cdot))>_{C(x, \eta)} \mid v \in W_{\#}^{1, p}\left(C(x, \eta), \mathbb{R}^{d}\right)\right\}
$$

from $\mathbb{R}^{n} \times \mathbb{R}^{n \times d}$ to $\mathbb{R}$, and the energy functional associated to its quasiconvex envelop $Q \mathcal{W}_{\eta, \epsilon}$

$$
I_{\eta, \epsilon}(u)=\int_{\Omega} Q \mathcal{W}_{\eta, \epsilon}(x, \nabla u), \text { for all } u \in W^{1, p}\left(\Omega, \mathbb{R}^{d}\right) .
$$

Remark 7 For $d=1$, quasiconvexification reduces to convexification and formula (13) is equivalent to formula (11).

Theorem 3 For $p>1$, the energy densities $Q \mathcal{W}_{\eta, \epsilon}$ are standard energy densities satisfying (12) and $I_{\eta, \epsilon} \Gamma\left(L^{p}\right)$ and $\Gamma\left(W^{1, p}\right)$-converges to $I_{h o m}$ as $\epsilon$ and $\eta$ go to 0 . Therefore, for any sequence $u_{\eta, \epsilon}$ of minimizers of $\inf \left\{I_{\eta, \epsilon}(v) \mid v \in W^{1, p}\left(\Omega, \mathbb{R}^{d}\right)+B C\right\}$, there exists a minimizer $u_{\text {hom }}$ of $\inf \left\{I_{h o m}(v) \mid v \in W^{1, p}\left(\Omega, \mathbb{R}^{d}\right)+B C\right\}$ such that

$$
\lim _{\eta \rightarrow 0} \lim _{\epsilon \rightarrow 0} u_{\eta, \epsilon}=u_{\text {hom }} \quad \text { weakly in } W^{1, p}\left(\Omega, \mathbb{R}^{d}\right),
$$

up to extraction.

Correspondingly, for any minimizer $u_{\text {hom }}$ of $\inf \left\{I_{h o m}(v) \mid v \in W^{1, p}\left(\Omega, \mathbb{R}^{d}\right)+B C\right\}$, there exists a sequence $u_{\eta, \epsilon}$ of minimizers of $\inf \left\{I_{\eta, \epsilon}(v) \mid v \in W^{1, p}\left(\Omega, \mathbb{R}^{d}\right)+B C\right\}$ such that

$$
\lim _{\eta \rightarrow 0} \lim _{\epsilon \rightarrow 0} u_{\eta, \epsilon}=u_{h o m} \quad \text { strongly in } W^{1, p}\left(\Omega, \mathbb{R}^{d}\right) .
$$

In addition, if $u_{\eta, \epsilon}$ is a family of equi isolated minimizers in the sense of Definition 4, then $u_{\text {hom }}$ is also isolated and (15) holds.

INRIA 
Theorem 3 is abstract since quasiconvexification is not explicit in general and very hard to compute in practice. However, an alternative consists in considering the energies $\mathcal{W}_{\eta, \epsilon}$ on a finite dimensional space first and passing to the limit on the dimension in a second step as stated in the following

Theorem 4 Let consider a set of finite dimensional subspaces of $W^{1, p}\left(\Omega, \mathbb{R}^{d}\right)$ and an associated equi-continuous family of projectors $\left(V_{H}, P_{H}\right)$, such that $V_{H^{1}} \subset V_{H^{2}}$ for $0 \leq H^{2} \leq H^{1}$ and ${\overline{\cup_{H} V_{H}}}^{1, p}=W^{1, p}(\Omega)$. We then define the following integral functionals on $W^{1, p}\left(\Omega, \mathbb{R}^{d}\right)$ :

$$
I_{\eta, \epsilon}^{H}(u)=\int_{\Omega} \mathcal{W}_{\eta, \epsilon}\left(x, \nabla P_{H} u\right)
$$

Under the assumptions of Theorem $3, I_{\eta, \epsilon}^{H} \Gamma\left(W^{1, p}\right)$-converges to $I_{h o m}$ as $\epsilon, \eta$ and $H$ go to 0 .

There exist minimizers $u_{\eta, \epsilon}^{H} \in V_{H}$ of $\inf \left\{I_{\eta, \epsilon}^{H}(v) \mid v \in W^{1, p}\left(\Omega, \mathbb{R}^{d}\right)+B C\right\}$, and for any such sequence there exists a minimizer $u_{\text {hom }}$ of $\inf \left\{I_{\text {hom }}(v) \mid v \in W^{1, p}\left(\Omega, \mathbb{R}^{d}\right)+B C\right\}$ such that

$$
\lim _{H \rightarrow 0} \lim _{\eta \rightarrow 0} \lim _{\epsilon \rightarrow 0} u_{\eta, \epsilon}^{H}=u_{\text {hom }} \quad \text { weakly in } W^{1, p}\left(\Omega, \mathbb{R}^{d}\right),
$$

up to extraction.

Correspondingly, for any minimizer $u_{\text {hom }}$ of $\inf \left\{I_{h o m}(v) \mid v \in W^{1, p}\left(\Omega, \mathbb{R}^{d}\right)+B C\right\}$, there exists a sequence $u_{\eta, \epsilon}^{H}$ of $\inf \left\{I_{\eta, \epsilon}^{H}(v) \mid v \in W^{1, p}\left(\Omega, \mathbb{R}^{d}\right)+B C\right\}$ in $V_{H}$, such that

$$
\lim _{H \rightarrow 0} \lim _{\eta \rightarrow 0} \lim _{\epsilon \rightarrow 0} u_{\eta, \epsilon}^{H}=u_{\text {hom }} \quad \text { strongly in } W^{1, p}\left(\Omega, \mathbb{R}^{d}\right) .
$$

In addition, if $u_{\eta, \epsilon}^{H}$ is a family of equi isolated minimizers in an extended sense of Definition 4 (for which $B$ shoud be replaced by $B \cap V_{H}$ ), then (18) holds.

The idea behind Theorem 4 is that the compactness of minimizers is due to the finite dimension of the minimization space at finite $\epsilon$ and $\eta$ and to quasiconvexity at the limit.

Remark 8 If $W$ is convex then, for all $\xi \in \mathbb{R}^{n \times d}$,

$$
\begin{aligned}
\inf \left\{\int_{(0,1)^{d}} W(\xi+\nabla u), u \in W_{\#}^{1, p}\left((0,1)^{d}, \mathbb{R}^{n}\right)\right\} \\
\quad=\inf \left\{\int_{(0,1)^{d}} W(\xi+\nabla u), u \in W^{1, p}\left((0,1)^{d}, \mathbb{R}^{n}\right),<\nabla u>_{(0,1)^{d}}=0\right\} .
\end{aligned}
$$

This equality does not hold for quasiconvex energy densities as can be easily seen on the polyconvex function $\xi \mapsto|\operatorname{det}(\xi)-1|$ on $\mathbb{R}^{2 \times 2}$ at point $\xi=0$. Therefore the averaged energy density of Definition 3 is not a suitable definition in the quasiconvex case, for which periodic or Dirichlet boundary conditions have to be considered.

$\mathrm{RR} \mathrm{n}^{\circ} 5791$ 
Remark 9 The convergence of the minimizers for quasiconvex energy densities in Theorem 3 is only weak in $W^{1, p}$, as opposed to the strictly convex case. This limitation is not technical and the hypothesis of "equi isolated minimizers" (which cannot be checked in practice) is in a way optimal. A counter example is given by shear band instabilities for a homogenized energy which is not strictly rank-one convex, as considered in [13] and numerically explored in [14].

\section{Proof of the main results}

For the sake of illustration, we first consider in Section 2.1 the one-dimensional linear version of (4). In this simple case, all the computations may be performed analytically. We thus get some useful insight on the interest of $u_{\eta, \epsilon}$ and on the ingredients needed for the proof of Theorem 1, which is in turn performed in Section 2.2 and adapted to the quasiconvex case in Section 2.3. The proof of Theorem 2 is then the purpose of Section 2.4.

\subsection{The one-dimensional linear case}

In the one-dimensional linear case, problem (4) reads

$$
\inf \left\{\int_{0}^{1} W_{\epsilon}\left(x, u^{\prime}(x)\right) d x, u \in H^{1}(0,1), u(0)=0, u(1)=1\right\},
$$

where $W_{\epsilon}(x, \xi)=\frac{1}{2} a_{\epsilon}(x) \xi^{2}$, and $a_{\epsilon}$ is a family of functions in $L^{\infty}(0,1)$ such that

$$
0<c \leq a_{\epsilon}(x) \leq C<+\infty
$$

for almost every $x \in \mathbb{R}$. To fix the ideas, we have made the boundary conditions specific in (19). This choice is arbitrary and plays no essential role.

The unique minimizer of (19) is:

$$
u_{\epsilon}(x)=C_{\epsilon} \int_{0}^{x} \frac{1}{a_{\epsilon}(y)} d y
$$

with $C_{\epsilon}=\left(\int_{0}^{1} \frac{1}{a_{\epsilon}(y)}\right)^{-1}$

Extracting a subsequence, we may assume that $\frac{1}{a_{\epsilon}}$ weakly converges to some $\frac{1}{b^{*}}$ in $L^{\infty}$. Consequently, $C_{\epsilon}$ converges to $C=\left(\int_{0}^{1} \frac{1}{b^{*}(x)} d x\right)^{-1}$ in $\mathbb{R}$. Thus $u_{\epsilon}$ weakly converges in $H^{1}(0,1)$ to $u_{h o m}: x \mapsto C \int_{0}^{x} \frac{1}{b^{*}(y)} d y$. 
In this one-dimensional case, the definition (6) of an averaged energy density reads

$$
\begin{array}{r}
W_{\eta, \epsilon}(x, \xi)=\frac{1}{2 \eta} \inf \left\{\int_{x-\eta}^{x+\eta} W_{\epsilon}\left(y, v^{\prime}(y)\right) d y, v \in H^{1}(x-\eta, x+\eta),\right. \\
\left.\frac{1}{2 \eta} \int_{x-\eta}^{x+\eta} v^{\prime}(y) d y=\xi\right\} .
\end{array}
$$

Straightforward calculations give the explicit form

$$
W_{\eta, \epsilon}(x, \xi)=\frac{1}{2}\left\langle\frac{1}{a_{\epsilon}(\cdot)}\right\rangle_{(x-\eta, x+\eta)}^{-1} \xi^{2} .
$$

It follows that the minimizer of

$$
\inf \left\{\int_{0}^{1} W_{\eta, \epsilon}\left(x, u^{\prime}(x)\right) d x, u \in H^{1}(0,1), u(0)=0, u(1)=1\right\}
$$

is the function

$$
u_{\eta, \epsilon}(x)=C_{\eta, \epsilon} \int_{0}^{x}\left\langle\frac{1}{a_{\epsilon}(\cdot)}\right\rangle_{(y-\eta, y+\eta)} d y
$$

where $C_{\eta, \epsilon}=\left(\int_{0}^{1}\left\langle\frac{1}{a_{\epsilon}(\cdot)}\right\rangle_{z-\eta, z+\eta} d z\right)^{-1}$. Let $C_{\eta}=\left(\int_{0}^{1}\left\langle\frac{1}{b^{*}(\cdot)}\right\rangle_{(z-\eta, z+\eta)} d z\right)^{-1}$. When $\eta$ is kept fixed, $u_{\eta, \epsilon}$ strongly converges in $H^{1}(0,1)$ (for the previous extraction in $\epsilon$ ) to $u_{\eta, h o m}(x)=C_{\eta} \int_{0}^{x}\left\langle\frac{1}{b^{*}(\cdot)}\right\rangle_{(y-\eta, y+\eta)} d y$ by the dominated convergence theorem. Let now $\eta$ go to zero. For every Lebesgue point $y$ of $\frac{1}{b^{*}} \in L^{1}(0,1)$ (thus almost everywhere on $(0,1)),\left\langle\frac{1}{b^{*}(\cdot)}\right\rangle_{(y-\eta, y+\eta)} \rightarrow \frac{1}{b^{*}(y)}$. The dominated convergence theorem then shows that $u_{\eta, h o m} \rightarrow u_{h o m}$ in $H^{1}(0,1)$. Consequently

$$
\lim _{\eta \rightarrow 0} \lim _{\epsilon \rightarrow 0} u_{\eta, \epsilon}=u_{h o m} \text { in } H^{1}(0,1) .
$$

Since the convergence obtained is strong in $H^{1}(0,1)$, the energy $\int_{0}^{1} W_{\eta, \epsilon}\left(x, u_{\eta, \epsilon}^{\prime}\right)$ also converges to $\int_{0}^{1} W_{h o m}\left(x, u_{\text {hom }}^{\prime}\right)$ in $\mathbb{R}$.

The convergence of $u_{\eta, \epsilon}$ to $u_{h o m}$ is strong, in contrast to that of $u_{\epsilon}$.

Remark 10 Remark 2 can be advantageously related to formula (20), where we can see that the compactness of translations allows to mix the limits in $\epsilon$ and $\eta$ as pointed out in [10] and illustrated below.

$\mathrm{RR} \mathrm{n}^{\circ} 5791$ 
Let us make the above one-dimensional problem even more specific by considering the example of an operator of the form $a_{\epsilon}(x)=a\left(\frac{x}{\epsilon}\right)$ where $a(\cdot)$ is 1-periodic on $\mathbb{R}$. In this case,

$$
\left\langle\frac{1}{a_{\epsilon}(\cdot)}\right\rangle_{(z-\eta, z+\eta)}=\frac{1}{2 \eta} \int_{z-\eta}^{z+\eta} \frac{1}{a(y / \epsilon)} d y=\frac{\epsilon}{2 \eta} \int_{\frac{z-\eta}{\epsilon}}^{\frac{z+\eta}{\epsilon}} \frac{1}{a(y)} d y=\left\langle\frac{1}{a(\cdot)}\right\rangle_{(0,1)}+O\left(\frac{\epsilon}{\eta}\right) .
$$

This shows that $\lim _{\epsilon \rightarrow 0} u_{\eta(\epsilon), \epsilon}=u_{\text {hom }}$ in $H^{1}(0,1)$ if $\lim _{\epsilon \rightarrow 0} \eta(\epsilon)=0$ and $\lim _{\epsilon \rightarrow 0} \frac{\epsilon}{\eta(\epsilon)}=0$, which is more precise than (21). The same property holds for stochastic homogenization for which we refer to the preliminaries and the Section 4 of [10].

In the proof of Theorem 1 for the one-dimensional case, the main ingredient is the pointwise convergence of integrands due to the averaging of weakly converging functions on balls. Since the expression of the minimizer in (20) is analytical, the conclusion is achieved by using the dominated convergence theorem. This specific expression is linked to the dimension and to the linearity. When dealing with the multi-dimensional case, no such analytical formula holds for the minimizer. Following the line of the proof for the onedimensional case, we can focus on the Green formula for the solution and use the abstract $G$-convergence theory. In the present work however, we focus on the minimum instead of the minimizer and use $\Gamma$-convergence arguments to link the convergence of the energies to the convergence of the minimizers. This approach illustrates that in a way the modified energy of Definition 3 is a relaxed energy, in the spirit of the homogenization of multiple integrals dealt with in [4].

\subsection{Proof of Theorem 1}

The following three lemmata relate the pointwise convergence of energy densities to the $\Gamma$-convergence of the associated energy functionals.

Lemma 6 [6, Proposition 5.11] Let $X=\mathbb{R}^{n}, B_{R}=B\left(x_{0}, R\right)$ for $x_{0} \in X$ and $R>0$, and let $F: B_{R} \rightarrow \mathbb{R}$ be a convex function. Suppose that $\sup _{x \in B_{R}} F(x)=M<+\infty$. Consequently $\inf _{x \in B_{R}} F(x)=m>-\infty$. Let $0<r<R$ and $K=(M-m) /(R-r)$. Then

$$
|F(x)-F(y)| \leq K\|x-y\|
$$

for every $x, y$ in the closure $\bar{B}_{r}$ of $B_{r}$.

Lemma 6 is a classical result of convex analysis. The reader is refered to [6] for a proof. It is used in the Appendix to prove the first of the following lemmata, which are crucial for the sequel, and also stated and proved in [6].

Lemma 7 [6, Proposition 5.14]. Let $\tilde{W}_{\epsilon}: \mathbb{R}^{n} \times \mathbb{R}^{n} \rightarrow \mathbb{R}$ be a set of Borel functions satisfying the growth condition

$$
0 \leq \tilde{W}_{\epsilon}(\cdot, \xi) \leq C\left(|\xi|^{p}+1\right)
$$


and such that for almost every $x \in \mathbb{R}^{n}, \tilde{W}_{\epsilon}(x, \cdot)$ is convex on $\mathbb{R}^{n}$. Let us assume that there exists an open bounded subset $\omega$ of $\mathbb{R}^{n}$, such that for all $\xi \in \mathbb{R}^{n}, \tilde{W}_{\epsilon}(\cdot, \xi)$ converges pointwise almost everywhere on $\omega$ to a function $\tilde{W}(\cdot, \xi)$. If $\tilde{W}$ is a Borel function that satisfies (23) and for almost every $x \in \omega, \tilde{W}(x, \cdot)$ is convex on $\mathbb{R}^{n}$, then $\tilde{I}_{\epsilon}: u \mapsto \int_{\omega} \tilde{W}_{\epsilon}(x, \nabla u) d x \Gamma\left(L^{p}\right)$ converges to $\tilde{I}: u \mapsto \int_{\omega} \tilde{W}(x, \nabla u) d x$ on $W^{1, p}(\omega)$.

Lemma 8 [6, Theorem 5.9]. Let $(X, d)$ be a metric space. Let $\left(F_{\epsilon}\right)$ be a sequence of functionals from $X$ to $\mathbb{R}$. If $\left(F_{\epsilon}\right)$ is equi-lower semicontinuous on $(X, d)$, then $F_{\epsilon} \Gamma(d)$-converges to $F$ in $X$ if and only if $F_{\epsilon}$ converges to $F$ pointwise in $X$.

Lemma 8 uses the notion of $\Gamma$-convergence in metric spaces. Provided the right extension of Definition 1 (see [5, Section 1.4] e.g.), its proof is rather direct. We refer to [6, Proposition $5.9]$ for details.

Finally we recall a particular case of a theorem of [6] which relates the convergence of minimizers to the $\Gamma$-convergence for non-coercive functionals. To this aim, let us introduce the following notions.

Definition 11 Let $F$ be a functional from the metric space $(X, d)$ to $\mathbb{R}$. We denote by $M(F)$ the possibly empty set of all the minimizers of $F$ on $X$. Let now $\left(F_{\epsilon}\right)$ be a sequence of functionals from $(X, d)$ to $\mathbb{R}$. We denote by $K-\lim _{\epsilon \rightarrow 0} M\left(F_{\epsilon}\right)$ the possibly empty set of the limits of all the sequences of minimizers $u_{\epsilon} \in M\left(F_{\epsilon}\right)$ in $(X, d)$.

We refer to [6, Sections 4 and 7$]$ for details on these notions and on the following

Lemma 9 [6, Theorem 7.19] Assume that $\left(F_{\epsilon}\right)$ is a sequence of functionals which $\Gamma(d)$ converges on the metric space $(X, d)$ to a functional $F$ that is not identically $+\infty$. If $\lim _{\epsilon \rightarrow 0} \inf _{X} F_{\epsilon}=\inf _{X} F$ and the infima are attained on $X$ (that is $M\left(F_{\epsilon}\right) \neq \emptyset$ ), then $M(F)=$ $\stackrel{\epsilon \rightarrow 0}{K}-\lim _{\epsilon \rightarrow 0} M\left(\stackrel{X}{F_{\epsilon}}\right)$. In particular, for any minimizer $u \in M(F)$, there exists a sequence $u_{\epsilon} \in M\left(F_{\epsilon}\right)$ such that $\lim _{\epsilon \rightarrow 0} u_{\epsilon}=u$ in $(X, d)$.

The sketch of the proof of the Theorem 1 is the following. We study separately the limits in $\epsilon$ and in $\eta$. The arguments are however the same. First, using Lemma 7 and the pointwise convergence of the integrand, we prove the $\Gamma\left(L^{p}\right)$-convergence of the sequence of functionals. Since these functionals are equi-coercive in the weak topology of $W^{1, p}(\Omega)$, Lemma 1 implies the convergence of the infima, and the existence and the weak convergence of the minimizers. In addition, the hypotheses of Lemma 8 are also satisfied on $\left(W^{1, p}(\Omega),\|\|_{W^{1, p}(\Omega)}\right)$, thus the sequence of functionals also $\Gamma\left(W^{1, p}\right)$-converges to the same limit. Finally we can apply Lemma 9 and deduce the strong convergence of the minimizers in the strictly convex case.

$\mathrm{RR} \mathrm{n}^{\circ} 5791$ 
Limit $\epsilon \rightarrow 0$ Up to extracting a subsequence in $\epsilon$ (not relabeled), Lemma 2, applied to $W_{\epsilon}$ on the open bounded subset $\cup_{x \in \Omega} B(x, \eta)$, implies that there exists an energy density $W_{h o m}$ whose associated energy $I_{h o m}$ is the $\Gamma$-limit of $I_{\epsilon}$ on $W^{1, p}\left(\cup_{x \in \Omega} B(x, \eta)\right)$. The locality of $\Gamma$-convergence (i.d. the energy density of the $\Gamma$-limit does not depend on the domain of integration), the irrelevance of boundary conditions for the $\Gamma$-convergence (Appendix 4.1) and the equi-coercivity (Lemma 1) imply

$$
\begin{aligned}
\lim _{\epsilon \rightarrow 0} & \left(\inf \left\{\int_{\omega} W_{\epsilon}(y, \nabla v) \mid v \in W^{1, p}(\omega),<\nabla v>_{\omega}=\xi\right\}\right) \\
\quad & \inf \left\{\int_{\omega} W_{h o m}(y, \nabla v) \mid v \in W^{1, p}(\omega),<\nabla v>_{\omega}=\xi\right\}
\end{aligned}
$$

for every open subset $\omega \subset \cup_{x \in \Omega} B(x, \eta)$ and for all $\xi \in \mathbb{R}^{n}$. Equality (24) with $\omega=B(x, \eta)$ reads

$$
\lim _{\epsilon \rightarrow 0} W_{\eta, \epsilon}(x, \xi)=W_{\eta, h o m}(x, \xi),
$$

for every $\xi \in \mathbb{R}^{n}$, where

$$
\begin{aligned}
W_{\eta, h o m}(x, \xi)=\inf \left\{<W_{h o m}(\cdot, \nabla v(\cdot))\right. & >_{B(x, \eta)} \mid \\
v & \left.\in W^{1, p}(B(x, \eta)),<\nabla v(\cdot)>_{B(x, \eta)}=\xi\right\} .
\end{aligned}
$$

The energy densities $W_{\eta, \epsilon}(\cdot, \xi)$ and $W_{\eta, h o m}(\cdot, \xi)$ are measurable for all $\epsilon$ and $\eta$ anf forall $\xi \in \mathbb{R}^{n}$ as the limits of the following measurable functions, $x \mapsto \int_{B(x, \eta)} W_{\epsilon}\left(y, \nabla u_{n}(y)\right)$ and $x \mapsto \int_{B(x, \eta)} W_{h o m}\left(y, \nabla v_{n}(y)\right)$ where $u_{n}$ and $v_{n}$ are minimizing sequences of these integrals on the set $\left\{v \in W^{1, p}(\Omega) \mid<\nabla v>_{B(x, \eta)}=\xi\right\}$. To prove that $W_{\eta, \epsilon}$ and $W_{\eta, h o m}$ are Carathéodory functions, it remains to prove that for almost every $x \in \Omega, W_{\eta, \epsilon}(x, \cdot)$ and $W_{\eta, h o m}(x, \cdot)$ are continuous on $\mathbb{R}^{n}$, which is actually a consequence of convexity (property H2).

We now show that the energy densities $W_{\eta, \epsilon}$ and $W_{\eta, h o m}$ satisfy the properties $H_{2}$ and H3. For almost every $x \in \Omega$ and for all $\xi \in \mathbb{R}^{n}$,

$$
\begin{aligned}
W_{\eta, \epsilon}(x, \xi) & =\inf \left\{<W_{\epsilon}(\cdot, \xi+\nabla v(\cdot))>_{B(x, \eta)} \mid<\nabla v>=0\right\} \\
& \leq<W_{\epsilon}(\cdot, \xi)>_{B(x, \eta)} \\
& \leq C\left(1+|\xi|^{p}\right), \quad \text { as } W_{\epsilon} \text { satisfies H3. }
\end{aligned}
$$

Let $v_{\xi}$ denote a minimizer of $\inf \left\{\left\langle W_{\epsilon}(\cdot, \xi+\nabla v(\cdot))>_{B(x, \eta)}\right|<\nabla v>_{B(x, \eta)}=0\right\}$, we then have

$$
\begin{aligned}
W_{\eta, \epsilon}(x, \xi) & \geq c<\left|\xi+\nabla v_{\xi}(\cdot)\right|^{p}>_{B(x, \eta)}, \quad \text { as } W_{\epsilon} \text { satisfies } H 3 \\
& \geq c \inf \left\{<|\xi+\nabla v(\cdot)|^{p}>_{B(x, \eta)} \mid<\nabla v>=0\right\} \\
& =c|\xi|^{p} .
\end{aligned}
$$


Consequently $W_{\eta, \epsilon}$ satisfies $H 3$. The same calculations hold for $W_{\eta, h o m}$. The convexity of $W_{\eta, \epsilon}(x, \cdot)$ and of $W_{\eta, h o m}(x, \cdot)$ is a consequence of the following calculation. Let $\xi, \zeta \in \mathbb{R}^{n}$, $\lambda \in(0,1)$ and $v_{1}, v_{2} \in W^{1, p}(\Omega)$ such that $<\nabla v_{1}>=<\nabla v_{2}>=0$. As $W_{\epsilon}$ is convex,

$$
\begin{aligned}
<W_{\epsilon}(\cdot, \lambda(\xi & \left.\left.+\nabla v_{1}(\cdot)\right)+(1-\lambda)\left(\zeta+\nabla v_{2}(\cdot)\right)\right)>_{B(x, \eta)} \\
& \leq \lambda<W_{\epsilon}\left(\cdot, \xi+\nabla v_{1}(\cdot)\right)>_{B(x, \eta)}+(1-\lambda)<W_{\epsilon}\left(\cdot, \zeta+\nabla v_{2}(\cdot)\right)>_{B(x, \eta)} .
\end{aligned}
$$

Let now $\tilde{v}_{1}$ and $\tilde{v}_{2}$ be respectively minimizers of the first and second terms of the right-hand side above. We have

$$
\begin{aligned}
&<W_{\epsilon}\left(\cdot, \lambda\left(\xi+\nabla \tilde{v}_{1}(\cdot)\right)+(1-\lambda)\right.\left.\left(\zeta+\nabla \tilde{v}_{2}(\cdot)\right)\right)>_{B(x, \eta)} \\
& \leq \lambda W_{\eta, \epsilon}(x, \xi)+(1-\lambda) W_{\eta, \epsilon}(x, \zeta)
\end{aligned}
$$

And consequently

$$
W_{\eta, \epsilon}(x, \lambda \xi+(1-\lambda) \zeta) \leq \lambda W_{\eta, \epsilon}(x, \xi)+(1-\lambda) W_{\eta, \epsilon}(x, \zeta) .
$$

It is worth noticing that if $W_{\epsilon}$ is strictly convex, then $W_{\eta, \epsilon}$ is also strictly convex.

Since $W_{\eta, \epsilon}$ and $W_{\eta, h o m}$ satisfy $H 1, H 2$ and $H 3$, we can use Lemma 7 and the pointwise convergence (25) to prove that

$$
\Gamma\left(L^{p}\right)-\lim _{\epsilon \rightarrow 0} I_{\eta, \epsilon}=I_{\eta, h o m}
$$

on $W^{1, p}(\Omega)$ where $I_{\eta, h o m}=u \mapsto \int_{\Omega} W_{\eta}(x, \nabla u)$. Using Lemma $1, H 3$ also implies the convergence of the infima

$$
\lim _{\epsilon \rightarrow 0} \inf \left\{I_{\eta, \epsilon}(u), u \in W^{1, p}(\Omega)+B C\right\}=\inf \left\{I_{\eta, h o m}(u), u \in W^{1, p}(\Omega)+B C\right\}
$$

and the weak convergence in $W^{1, p}(\Omega)$ of any corresponding sequence of minimizers $u_{\eta, \epsilon}$ to some minimizer $u_{\eta, h o m}$, up to extraction.

In addition, by the application of the dominated convergence theorem, for every $u \in$ $W^{1, p}(\Omega), \int_{\Omega} W_{\eta, \epsilon}(x, \nabla u)$ converges to $\int_{\Omega} W_{\eta, h o m}(x, \nabla u)$ as $\epsilon$ goes to 0 . As $\left(W_{\eta, \epsilon}\right)$ is convex and satisfies $H 3$ for all $\epsilon$ and $\eta$, the associated energy functionals are equi continuous on $W^{1, p}(\Omega)$. Thus Lemma 8 shows that $I_{\eta, \epsilon} \Gamma\left(W^{1, p}\right)$-converges to $I_{\eta, \text { hom }}$.

Lemma 9 shows that for every minimizer $u_{\eta, h o m}$ there exists a sequence of minimizers $u_{\eta, \epsilon}$ such that $u_{\eta, \epsilon} \rightarrow u_{\eta, h o m}$ in $W^{1, p}(\Omega)$. In addition, if $W_{\epsilon}$ is strictly convex, then $W_{\eta, \epsilon}$ is also strictly convex and there exists a unique sequence of minimizers $u_{\eta, \epsilon}$. As $M\left(I_{\eta, h o m}\right)$ is non empty, $\left(u_{\eta, \epsilon}\right)$ strongly converges to some $u_{\eta, h o m} \in M\left(I_{\eta, h o m}\right)$ (without extraction) in $W^{1, p}(\Omega)$ as $\epsilon \rightarrow 0$, and the minimizer $u_{\eta, h o m}$ is also unique.

If a sequence of minimizers $u_{\eta, \epsilon}$ happens to be equi isolated on $B \subset W^{1, p}(\Omega)$ in the sense of Definition 4 , then the previous argument holds applying Lemma 9 on $\left(B,\|\cdot\|_{1, p}\right)$ since the $\Gamma\left(W^{1, p}\right)$-convergence on $W^{1, p}(\Omega)$ implies the $\Gamma\left(W^{1, p}\right)$-convergence on $\left(B,\|\cdot\|_{1, p}\right)$. We thus obtain the strong convergence of the sequence.

$\mathrm{RR} \mathrm{n}^{\circ} 5791$ 
Limit $\eta \rightarrow 0 \quad$ For every $\xi \in \mathbb{R}^{n}$, let us first determine the pointwise limit on $\Omega$ as $\eta$ goes to 0 of

$$
\begin{gathered}
W_{\eta, h o m}(x, \xi)=\inf \left\{<W_{h o m}(\cdot, \nabla v(\cdot))>_{B(x, \eta)} \mid v \in W^{1, p}(B(x, \eta)),<\nabla v>_{B(x, \eta)}=\xi\right\} \\
=\inf \left\{\int_{B(0,1)} W_{h o m}(x+\eta y, \nabla v(y)), v \in W^{1, p}(B(0,1)),\right. \\
\left.\quad<\nabla v>_{B(0,1)}=\xi\right\} \cdot \frac{1}{\mathcal{L}^{n}(B(0,1))}
\end{gathered}
$$

To this end, let denote by $\tilde{W}_{x}^{\eta}(y, \xi)=W_{h o m}(x+\eta y, \xi)$ for almost every $x \in \Omega, y \in B(0,1)$ and $\xi \in \mathbb{R}^{n}$. Lemma 2 implies that the energy densities $\tilde{W}_{x}^{\eta}(\cdot, \cdot)$ and $W_{h o m}(x, \cdot)$ satisfy $H 1$, H2 and H3. In addition, for all $\xi \in \mathbb{R}^{n}$, every Lebesgue point $x \in \Omega$ (and consequently almost everywhere on $\Omega$ ) of $W_{\text {hom }}(\cdot, \xi) \in L^{1}(\Omega, \mathbb{R})$ and almost every $y \in B(0,1)$,

$$
\lim _{\eta \rightarrow 0} W_{\text {hom }}(x+\eta y, \xi)=W_{\text {hom }}(x, \xi) .
$$

We now apply Lemma 7 to obtain the $\Gamma\left(L^{p}\right)$-convergence of the associated integral functionals. Property H3 then implies the convergence of the infima (26) by the application of Lemma 1. For every $\xi \in \mathbb{R}^{n}$, this proves the following pointwise convergence almost everywhere on $\Omega$

$$
\lim _{\eta \rightarrow 0} W_{\eta, h o m}(x, \xi)=W_{h o m}(x, \xi) .
$$

Lemma 2 and the same arguments as for $W_{\eta, \epsilon}$ show that $W_{\eta, h o m}$ and $W_{h o m}$ also satisfy H1, H2 and H3. By the application of Lemma 7, the pointwise convergence (27) implies the $\Gamma\left(L^{p}\right)$-convergence of $I_{\eta, h o m}$ to $I_{h o m}$ on $W^{1, p}(\Omega)$ as $\eta$ goes to 0 . Consequently, minimizers $u_{\eta, h o m}$ converge weakly in $W^{1, p}(\Omega)$ to some minimizer $u_{\text {hom }}$, up to extraction, by Lemma 1 . Using Lemmata 8 and 9 as for the limit $\epsilon \rightarrow 0$, we obtain the three last statements of Theorem 1.

\subsection{Proof of Theorem 3}

The proof of Theorem 3 exactly follows the lines of the proof of Theorem 1 . We indeed have

Remark 11 The conclusion of Lemma 7 is unchanged if we replace the hypothesis of convexity on $\mathbb{R}^{n}$ by the hypothesis of quasiconvexity on $\mathbb{R}^{n \times d}$.

This version of Lemma 7 is more general and is the one proved in the Appendix. For the sake of clarity, we will still refer to Lemma 7 , even in the quasiconvex setting for which it should be understood in the sense of Remark 11.

In contrast to the proof of Theorem 1, the hypotheses of Lemmata 7 and 8 are more technical to check in the quasiconvex case, especially the pointwise convergence of the integrands. To do that, we will make use of the following results of the calculus of variations: a characterization of quasiconvex hulls and a decomposition lemma.

INRIA 
Lemma 10 [2, III.7] Let $f$ be a Carathéodory functional satisfying the growth condition (12) for $p>1$ on $\mathbb{R}^{n} \times \mathbb{R}^{n \times d}$. Let $Q f$ denotes the quasiconvex envelop of $f$. Then for all $\Omega$ open bounded subset of $\mathbb{R}^{n}$ and for all $u \in W^{1, p}\left(\Omega, \mathbb{R}^{d}\right)$, there exists a sequence $\left\{\phi_{k}\right\}_{k} \in$ $W^{1, p}\left(\Omega, \mathbb{R}^{d}\right)$ such that $\phi_{k} \rightarrow u$ in $W^{1, p}\left(\Omega, \mathbb{R}^{d}\right)$ and

$$
\int_{\Omega} Q f(x, \nabla u)=\lim _{k \rightarrow \infty} \int_{\Omega} f\left(x, \nabla \phi_{k}\right) .
$$

Lemma 11 [12] Let $p>1$ and assume that $\partial \Omega$ is Lipschitz. Let $u_{k} \rightarrow v_{0}$ in $W^{1, p}\left(\Omega, \mathbb{R}^{d}\right)$. Then there exists a subsequence $u_{k_{l}}$ of $u_{k}$ and a sequence $v_{l} \in W^{1, \infty}\left(\mathbb{R}^{n}, \mathbb{R}^{d}\right)$ such that

(i) $v_{l} \rightarrow v_{0}$ in $W^{1, p}\left(\Omega, \mathbb{R}^{d}\right)$,

(ii) $v_{l}=v_{0}$ in a neighborhood of $\partial \Omega$,

(iii) $\left\{\nabla v_{l}\right\}_{l}$ is p-equi-integrable, that is for all $\rho>0$, there exists $\delta>0$ such that for all measurable subset $A \subset \Omega, \sup _{l \in \mathbb{N}} \int_{A}\left|\nabla v_{l}\right|^{p} d x<\rho$ whenever $\mathcal{L}^{n}(A)<\delta$,

(iv) $\lim _{l \rightarrow \infty} \mathcal{L}^{n}\left(\left\{x \in \Omega: v_{l}(x) \neq u_{k_{l}}(x)\right.\right.$ or $\left.\left.\nabla v_{l}(x) \neq \nabla u_{k_{l}}(x)\right\}\right)=0$.

We finally state a lemma that relates the pointwise convergence of Lipschitz Carathéodory functions to the pointwise convergence of their quasiconvex envelops. The proof of this lemma is posponed until the Appendix.

Lemma 12 Let $f$ and $f_{\epsilon}$ be Carathéodory functions satisfying (12) on $\mathbb{R}^{n} \times \mathbb{R}^{n \times d}$. We assume that for all $R>0$, there exists $K>0$ such that for almost every $x \in \mathbb{R}^{n}$ and for all $\epsilon>0, f_{\epsilon}(x, \cdot)$ and $f$ are K-Lipschitz on $B(0, R)=\left\{\xi \in \mathbb{R}^{n \times d}:|\xi| \leq R\right\}$. If $f_{\epsilon}(x, \xi)$ converges to $f(x, \xi)$ for almost every $x \in \mathcal{O}$ (open bounded subset of $\mathbb{R}^{n}$ with Lipschitz boundary $\partial \mathcal{O})$ and for all $\xi \in \mathbb{R}^{n \times d}$, then $Q f_{\epsilon}(x, \xi)$ converges to $Q f(x, \xi)$ for almost every $x \in \mathcal{O}$ and for all $\xi \in \mathbb{R}^{n \times d}$.

We are now in position to prove Theorems 3 and 4 . We only treat the limit $\epsilon \rightarrow 0$, the one for $\eta$ being essentially the same as for $\epsilon$ provided the same adaptations as in Section 2.2.

Proof of Theorem 3 We first introduce the following energy density defined for almost every $x \in \mathbb{R}^{n}$ and for all $\xi \in \mathbb{R}^{n \times d}$ by

$$
\mathcal{W}_{\eta, h o m}(x, \xi)=\inf \left\{<W_{h o m}(y, \xi+\nabla v)>_{C(x, \eta)} \mid v \in W_{\#}^{1, p}\left(C(x, \eta), \mathbb{R}^{d}\right)\right\} .
$$

Arguing as for the previous Section, we can prove that $\mathcal{W}_{\eta, \epsilon}$ and $\mathcal{W}_{\eta, h o m}$ satisfy (H2) and (H3). Thus $Q \mathcal{W}_{\eta, \epsilon}$ and $Q \mathcal{W}_{\eta, h o m}$ are standard energy densities satisfying (12) with the same coefficients as for $W_{\epsilon}$. Provided that

$$
\lim _{\epsilon \rightarrow 0} Q \mathcal{W}_{\eta, \epsilon}(x, \xi)=Q \mathcal{W}_{\eta, h o m}(x, \xi)
$$


for almost every $x \in \mathbb{R}^{n}$ and for all $\xi \in \mathbb{R}^{n \times d}$, Lemma 7 implies the $\Gamma\left(L^{p}\right)$-convergence of $I_{\eta, \epsilon}$ to $I_{\eta, h o m}$ on $W^{1, p}\left(\Omega, \mathbb{R}^{d}\right)$, defined by

$$
I_{\eta, h o m}(u)=\int_{\Omega} Q \mathcal{W}_{\eta, \mathrm{hom}}(x, \nabla u) .
$$

Let us prove the pointwise convergence (29). The energy densities $\mathcal{W}_{\eta, \epsilon}$ and $\mathcal{W}_{\eta, h o m}$ actually satisfy the hypotheses of Lemma 12 .

Arguing as in Section 2.2, we have

- $\lim _{\epsilon \rightarrow 0} \mathcal{W}_{\eta, \epsilon}(x, \xi)=\mathcal{W}_{\eta, h o m}(x, \xi)$ for almost every $x \in \mathbb{R}^{n}$ and for all $\xi \in \mathbb{R}^{n \times d}$,

- $\mathcal{W}_{\eta, \epsilon}$ and $\mathcal{W}_{\eta, h o m}$ satisfy (12).

It remains to prove that $\mathcal{W}_{\eta, \epsilon}(x, \cdot)$ and $\mathcal{W}_{\eta, h o m}(x, \cdot)$ are equi locally Lipschitz (and thus continuous) on $\mathbb{R}^{n \times d}$ to fullfil the assumptions of Lemma 12 .

Let us recall that standard energy densities $W$ are locally Lipschitz in the following sense: there exists $\mathcal{C}>0$ only depending on $p, c$ and $C$ in (12) such that for almost every $x \in \mathbb{R}^{n}$ and for all $\xi_{1}, \xi_{2} \in \mathbb{R}^{n \times d}$,

$$
\left|W\left(x, \xi_{1}\right)-W\left(x, \xi_{2}\right)\right| \leq \mathcal{C}\left(1+\left|\xi_{1}\right|^{p-1}+\left|\xi_{2}\right|^{p-1}\right)\left|\xi_{1}-\xi_{2}\right| .
$$

Convex functions such that (12) holds satisfy inequality (30) using Lemma 6. It can be proved for rank-one convex functions (and thus for quasiconvex functions) by introducing a decomposition of $\xi_{1}-\xi_{2}$ in a sum of rank-one matrices.

For every fixed $x \in \mathbb{R}^{n}$, let $u_{\xi_{1}}$ and $u_{\xi_{2}}$ be minimizers of (28) for $\mathcal{W}_{\eta, \epsilon}$ and $\xi=\xi_{1}$ and $\xi_{2}$ respectively. The following four inequalities hold:

$$
\begin{aligned}
& \mathcal{W}_{\eta, \epsilon}\left(x, \xi_{1}\right)-\frac{1}{\eta^{n}} \int_{C(x, \eta)} W_{\epsilon}\left(y, \nabla u_{\xi_{2}}+\xi_{1}\right) \leq 0, \\
& \mathcal{W}_{\eta, \epsilon}\left(x, \xi_{2}\right)-\frac{1}{\eta^{n}} \int_{C(x, \eta)} W_{\epsilon}\left(y, \nabla u_{\xi_{1}}+\xi_{2}\right) \leq 0, \\
& \left|\mathcal{W}_{\eta, \epsilon}\left(x, \xi_{1}\right)-\frac{1}{\eta^{n}} \int_{C(x, \eta)} W_{\epsilon}\left(y, \nabla u_{\xi_{1}}+\xi_{2}\right)\right| \leq C\left(1+\left|\xi_{1}\right|^{p-1}+\left|\xi_{2}\right|^{p-1}\right)\left|\xi_{1}-\xi_{2}\right|, \\
& \left|\mathcal{W}_{\eta, \epsilon}\left(x, \xi_{2}\right)-\frac{1}{\eta^{n}} \int_{C(x, \eta)} W_{\epsilon}\left(y, \nabla u_{\xi_{2}}+\xi_{1}\right)\right| \leq C\left(1+\left|\xi_{1}\right|^{p-1}+\left|\xi_{2}\right|^{p-1}\right)\left|\xi_{1}-\xi_{2}\right| .
\end{aligned}
$$

The first two inequalities of (31) are direct consequences of the definitions of $u_{\xi_{1}}$ and $u_{\xi_{2}}$. The last two inequalities are obtained by integrating (30) over $C(x, \eta)$ with $W=W_{\epsilon}$ and noting that (12) implies $\int_{C(x, \eta)}\left|\nabla u_{\xi_{1}}\right|^{p} \leq p(C+1)\left(1+\left|\xi_{1}\right|^{p}\right) \eta^{n}$ by the triangle inequality (see the proof of (47) in Section 2.4 for details). The combination of these four inequalities 
shows that $\mathcal{W}_{\eta, \epsilon}$ satisfies (30) for some $\mathcal{C}>0$ which is independent of $\epsilon$ and $\eta$. The same result and proof hold for $\mathcal{W}_{\eta, h o m}$.

Lemma 12 then implies the pointwise convergence (29). We can obtain the $\Gamma\left(L^{p}\right)$ convergence of $I_{\eta, \epsilon}$ to $I_{\eta, h o m}$ by applying Lemma 7. The functionals $Q \mathcal{W}_{\eta, \epsilon}$ and $Q \mathcal{W}_{\eta, h o m}$ are equi locally bounded by (12), and thus equi locally Lipschitz and equi continuous on $W^{1, p}\left(\Omega, \mathbb{R}^{d}\right)$. Therefore, as in Section 2.2 , Lemma 8 proves the $\Gamma\left(W^{1, p}\right)$-convergence of these energies. From Lemma 9 we then deduce the existence of strong converging sequences of minimizers, and the strong convergence of sequences of equi isolated minimizers if they exist.

Proof of Theorem 4 The proof of Theorem 4 is based on two arguments. At fixed $H$, the energies are equi locally Lipschitz because of the equi continuity of the projections $P_{H}$. Therefore the pointwise convergence on $W^{1, p}(\Omega)$ of $I_{\eta, \epsilon}^{H}$ to $I_{h o m}^{H}$, which is a consequence of the dominated convergence theorem, implies the $\Gamma\left(W^{1, p}\right)$-convergence of the energy functionals at fixed $H$ when $\epsilon$ and $\eta$ go to 0 .

In addition, the infimum $\inf \left\{I_{\eta, \epsilon}^{H}(v), v \in W^{1, p}\left(\Omega, \mathbb{R}^{d}\right)+B C\right\}$ is attained on $V_{H}$. It suffices indeed to consider the projection of any minimizing sequence in $W^{1, p}\left(\Omega, \mathbb{R}^{d}\right)$ on $V_{H}$. This new sequence is still a minimizing sequence since the energy is not changed by the projection and it is bounded in the finite dimensional space $V_{H}$. Therefore it converges up to extraction in $\left(V_{H},\|\cdot\|_{1, p}\right)$. The continuity of the energy (it is locally Lipschitz) then allows to pass to the limit and proves the existence of minimizers $u_{\eta, \epsilon}^{H} \in V_{H}$ of $\inf \left\{I_{\eta, \epsilon}^{H}(v), v \in W^{1, p}\left(\Omega, \mathbb{R}^{d}\right)+B C\right\}$. A following direct argument also shows the convergence of the infima

$$
\lim _{\eta \rightarrow 0} \lim _{\epsilon \rightarrow 0} \inf \left\{I_{\eta, \epsilon}^{H}(v), v \in W^{1, p}\left(\Omega, \mathbb{R}^{d}\right)+B C\right\}=\inf \left\{I_{h o m}^{H}(v), v \in W^{1, p}\left(\Omega, \mathbb{R}^{d}\right)+B C\right\} .
$$

Let us detail this direct argument by considering a sequence $u_{\eta, \epsilon}^{H} \in V_{H}$ of minimizers of $\inf \left\{I_{\eta, \epsilon}^{H}(v), v \in W^{1, p}\left(\Omega, \mathbb{R}^{d}\right)+B C\right\}$. This sequence is bounded in $W^{1, p}\left(\Omega, \mathbb{R}^{d}\right)$ using the growth condition (12) and thus compact in $W^{1, p}(\Omega)$ since $V_{H}$ is finite dimensional. Therefore there exists $u_{h o m}^{H} \in V_{H}$ such that $\lim _{\eta \rightarrow 0} \lim _{\epsilon \rightarrow 0} u_{\eta, \epsilon}^{H}=u_{h o m}^{H}$ in $W^{1, p}(\Omega)$. We then have

$$
\left|I_{\eta, \epsilon}^{H}\left(u_{\eta, \epsilon}^{H}\right)-I_{h o m}^{H}\left(u_{h o m}^{H}\right)\right| \leq\left|I_{\eta, \epsilon}^{H}\left(u_{\eta, \epsilon}^{H}\right)-I_{\eta, \epsilon}^{H}\left(u_{h o m}^{H}\right)\right|+\left|I_{\eta, \epsilon}^{H}\left(u_{h o m}^{H}\right)-I_{h o m}^{H}\left(u_{h o m}^{H}\right)\right| .
$$

The second term of the right-hand side goes to 0 thanks to the pointwise convergence of the energy as $\epsilon$ and $\eta$ go to 0 , whereas the first term goes to 0 thanks to the equi local Lipchitz property of $I_{\eta, \epsilon}^{H}$ :

$$
\left|I_{\eta, \epsilon}^{H}\left(u_{\eta, \epsilon}^{H}\right)-I_{\eta, \epsilon}^{H}\left(u_{h o m}^{H}\right)\right| \leq C\left(1+\left\|\nabla u_{\eta, \epsilon}^{H}\right\|_{p}^{p-1}+\left\|\nabla u_{h o m}^{H}\right\|_{p}^{p-1}\right)\left\|\nabla u_{\eta, \epsilon}^{H}-\nabla u_{h o m}^{H}\right\|_{p},
$$

which also vanishes since $u_{\eta, \epsilon}^{H}$ converges to $u_{h o m}^{H}$ in $W^{1, p}\left(\Omega, \mathbb{R}^{d}\right)$. It remains to prove that $u_{h o m}^{H}$ is a minimizer of $\inf \left\{I_{h o m}^{H}(v), v \in W^{1, p}\left(\Omega, \mathbb{R}^{d}\right)+B C\right\}$. For all $v \in W^{1, p}\left(\Omega, \mathbb{R}^{d}\right)$, for all $\eta$ and $\epsilon, I_{\eta, \epsilon}^{H}\left(u_{\eta, \epsilon}^{H}\right) \leq I_{\eta, \epsilon}^{H}(v)$. At the limit, we obtain $I_{h o m}^{H}\left(u_{h o m}^{H}\right) \leq I_{h o m}^{H}(v)$, which proves the statement since $v$ is arbitrary.

$\mathrm{RR} \mathrm{n}^{\circ} 5791$ 
At fixed $H$, we have proved the $\Gamma\left(W^{1, p}\right)$-convergence of $I_{\eta, \epsilon}^{H}$ to $I_{h o m}^{H}$ on $W^{1, p}\left(\Omega, \mathbb{R}^{d}\right)$ when $\epsilon$ and $\eta$ go to 0 , and the existence and the strong convergence of any sequence of minimizers $u_{\eta, \epsilon}^{H} \in V_{H}$ of $\inf \left\{I_{\eta, \epsilon}^{H}(v), v \in W^{1, p}\left(\Omega, \mathbb{R}^{d}\right)+B C\right\}$ to some minimizer $u_{h o m}^{H} \in V_{H}$ of $\inf \left\{I_{h o m}^{H}(v), v \in W^{1, p}\left(\Omega, \mathbb{R}^{d}\right)+B C\right\}$, up to extraction.

The limit $H \rightarrow 0$ can be dealt with as follows. Since the sequence $u_{\text {hom }}^{H}$ is bounded in $W^{1, p}(\Omega)$ by the growth condition (12), we can extract a subsequence (not relabeled) weakly converging to some $u_{h o m} \in W^{1, p}(\Omega)$. We now prove that $u_{h o m}$ is a minimizer of $\inf \left\{I_{h o m}(v), v \in W^{1, p}\left(\Omega, \mathbb{R}^{d}\right)+B C\right\}$ and that $I_{\text {hom }}^{H}\left(u_{\text {hom }}^{H}\right) \rightarrow I_{\text {hom }}\left(u_{\text {hom }}\right)$. The quasiconvexity of $W_{h o m}$ and (12) imply the lower semi-continuity of $I_{h o m}$ for the weak topology of $W^{1, p}(\Omega)$, which shows

$$
I_{\text {hom }}\left(u_{\text {hom }}\right) \leq \lim _{H \rightarrow 0} I_{\text {hom }}\left(u_{\text {hom }}^{H}\right) .
$$

Next, as a consequence of the continuity of $I_{h o m}$ for the strong topology of $W^{1, p}(\Omega)$, for all $v \in W^{1, p}(\Omega)+B C$ and for all $\rho>0$ there exists $H>0$ and $v_{H} \in V_{H}+B C$ such that $I_{h o m}(v) \geq I_{h o m}\left(v_{H}\right)-\rho \geq I_{h o m}\left(u_{h o m}^{H}\right)-\rho \geq \lim _{H \rightarrow 0} I_{h o m}\left(u_{h o m}^{H}\right)-\rho$, which exists as a decreasing sequence of positive numbers. Thus $u_{h o m}$ is a minimizer of $I_{h o m}$ on $W^{1, p}(\Omega)+B C$.

The convergence of the infima is also obtained by this continuity argument. The pointwise convergence of the sequence $I_{h o m}^{H}$ and its equi local Lipschitz property allow to use Lemma 8 and show the $\Gamma\left(W^{1, p}\right)$-convergence of $I_{h o m}^{H}$ to $I_{h o m}$ on $W^{1, p}(\Omega)$. Using the convergence of the infima, we can then apply Lemma 9 and prove that, with obvious notation, for any minimizer $u_{\text {hom }} \in W^{1, p}(\Omega)$ there exists a sequence of minimizers $u_{h o m}^{H} \in W^{1, p}(\Omega)$ such that $\lim _{H \rightarrow 0} u_{\text {hom }}^{H}=u_{\text {hom }}$ in $W^{1, p}(\Omega)$. These minimizers may not belong to any $V_{H}$. Moreover, due to the projection $P_{H}$ in the energy, the minimizers of $\inf \left\{I_{h o m}^{H}(v), v \in W^{1, p}\left(\Omega, \mathbb{R}^{d}\right)+B C\right\}$ are never isolated, therefore the argument used to prove the strong convergence in Theorem 3 has to be slightly modified to apply here.

To this aim, let consider a sequence of minimizers $u_{\text {hom }}^{H} \in V_{H}$ which is equi isolated in the following extended sense. We suppose that there exists a ball $B \subset W^{1, p}(\Omega)$ such that $u_{h o m}^{H}$ is the unique minimizer of $\inf \left\{I_{h o m}^{H}(v), v \in W^{1, p}\left(\Omega, \mathbb{R}^{d}\right)+B C\right\}$ on $B \cap V_{H}$. Let $u_{\text {hom }}$ be a weak limit of this sequence. Because of the weakly lower semicontinuity of the norm, $u_{\text {hom }} \in B$. In addition, there exists another sequence $v_{\text {hom }}^{H} \in W^{1, p}(\Omega)$ of minimizers of $\inf \left\{I_{h o m}^{H}(v), v \in W^{1, p}\left(\Omega, \mathbb{R}^{d}\right)+B C\right\}$ such that $v_{h o m}^{H} \rightarrow u_{\text {hom }}$ in $W^{1, p}(\Omega)$. Because of this strong convergence, it is not restrictive to suppose $v_{\text {hom }}^{H} \in B$, and $P_{H}\left(v_{\text {hom }}^{H}\right) \in B$ as well. Since $P_{H}\left(v_{h o m}^{H}\right) \in B$ is a minimizer of $\inf \left\{I_{h o m}^{H}(v), v \in W^{1, p}\left(\Omega, \mathbb{R}^{d}\right)+B C\right\}$ and the sequence $u_{\text {hom }}^{H}$ is equi isolated in the sense above, $u_{h o m}^{H}=P_{H}\left(v_{h o m}^{H}\right)$.

By the triangle inequality we then have $\left\|u_{\text {hom }}^{H}-u_{\text {hom }}\right\|_{1, p} \leq\left\|P_{H}\left(v_{h o m}^{H}-u_{\text {hom }}\right)\right\|_{1, p}+$ $\left\|P_{H}\left(u_{\text {hom }}\right)-u_{\text {hom }}\right\|_{1, p}$. The first term of the right hand-side goes to 0 because of the equi continuity of the family of projections $\left(P_{H}\right)$ and the strong convergence of $v_{h o m}^{H}$ to $u_{h o m}$, whereas the second term goes to 0 by definition of the family of spaces $V_{H}$. This shows the strong convergence of $u_{h o m}^{H}$ and concludes the proof of the theorem. 


\section{$2.4 \quad$ Proof of Theorem 2}

The proof of Theorem 2 extensively uses the following consequences of the properties of equi-continuity (2) and equi-monotonicity (3) of the elliptic operators.

Lemma 13 We keep the notation of Lemma 3. For almost all $x \in \Omega$ and for all $\xi_{1}, \xi_{2} \in \mathbb{R}^{n}$, property (2) implies

$$
\left|W_{\epsilon}\left(x, \xi_{1}\right)-W_{\epsilon}\left(x, \xi_{2}\right)\right| \leq C\left|\xi_{1}-\xi_{2}\right|\left(1+\left|\xi_{1}\right|^{p-1}+\left|\xi_{2}\right|^{p-1}\right) .
$$

For all convex subset $K \subset W^{1, p}(\Omega)$, if u minimizes $\inf \left\{\int_{\Omega} W_{\epsilon}(x, \nabla v) \mid v \in K\right\}$, then property (3) implies

$$
\left|\int_{\Omega}\left(W_{\epsilon}(\nabla u)-W_{\epsilon}(\nabla v)\right)\right| \geq c\|\nabla u-\nabla v\|_{L^{p}(\Omega)}^{p}
$$

for all $v \in K$ if $\beta \leq p$.

The proof of Lemma 13 is classical (see [19]) and postponed until the Appendix.

Let us introduce:

$$
\begin{aligned}
u_{\epsilon} & =\operatorname{Argmin}\left\{\int_{\Omega}\left(W_{\epsilon}(x, \nabla u)-f u\right) \mid u \in W^{1, p}(\Omega)+B C\right\} \\
u_{h o m} & =\operatorname{Argmin}\left\{\int_{\Omega}\left(W_{h o m}(x, \nabla u)-f u\right) \mid u \in W^{1, p}(\Omega)+B C\right\} \\
v_{\eta, \epsilon} & =\operatorname{Argmin}\left\{\int_{\Omega}\left(W_{\eta, \epsilon}(x, \nabla u)-f u\right) \mid u \in W^{1, p}(\Omega)+B C\right\} \\
u_{\epsilon}^{H, i} & =\operatorname{Argmin}\left\{\int_{Q_{H, i}} W_{\epsilon}(x, \nabla u) \mid<\nabla u>_{Q_{H, i}}=<\nabla u_{\epsilon}>_{Q_{H, i}}\right\} \\
v_{\eta, \epsilon}^{H, i} & =\operatorname{Argmin}\left\{\int_{Q_{H, i}} W_{\epsilon}(x, \nabla u) \mid<\nabla u>_{Q_{H, i}}=<\nabla v_{\eta, \epsilon}>_{Q_{H, i}}\right\} \\
u_{h o m}^{H, i} & =\operatorname{Argmin}\left\{\int_{Q_{H, i}} W_{\epsilon}(x, \nabla u) \mid<\nabla u>_{Q_{H, i}}=<\nabla u_{h o m}>_{Q_{H, i}}\right\}
\end{aligned}
$$

Let denote by $I_{H}$ the cardinal of the partition of $\Omega$. We have

$$
\begin{aligned}
\int_{\Omega}\left|\nabla u_{\epsilon}-\sum_{i=1}^{I_{H}} \nabla v_{\eta, \epsilon}^{H, i} 1_{Q_{H, i}}\right|^{p}=\sum_{i}^{I_{H}} \int_{Q_{H, i}}\left|\nabla u_{\epsilon}-\nabla v_{\eta, \epsilon}^{H, i}\right|^{p} \\
\leq p \sum_{i=1}^{I_{H}}\left(\int_{Q_{H, i}}\left|\nabla u_{\epsilon}-\nabla u_{\epsilon}^{H, i}\right|^{p}+\int_{Q_{H, i}}\left|\nabla u_{\epsilon}^{H, i}-\nabla v_{\eta, \epsilon}^{H, i}\right|^{p}\right) \\
=p \sum_{i=1}^{I_{H}}\left(A_{i}^{\epsilon, H}+B_{i}^{\epsilon, H, \eta}\right)
\end{aligned}
$$

$\mathrm{RR} \mathrm{n}^{\circ} 5791$ 
We now separately examine the limit $(H, \eta, \epsilon) \rightarrow 0$ in the two sums $\sum_{i=1}^{I_{H}} A_{i}^{\epsilon, H}$ and $\sum_{i=1}^{I_{H}} B_{i}^{\epsilon, H, \eta}$.

We show that both terms vanish in the limit in the following sense:

$$
\begin{aligned}
& \lim _{H \rightarrow 0} \limsup _{\epsilon \rightarrow 0} \sum_{i=1}^{I_{H}} A_{i}^{\epsilon, H}= 0, \\
& \lim _{\eta \rightarrow 0} \limsup _{\epsilon \rightarrow 0} \sum_{i=1}^{I_{H}} B_{i}^{\epsilon, H, \eta}=0, \quad \text { uniformly in } H .
\end{aligned}
$$

Limit of $\sum_{i=1}^{I_{H}} A_{i}^{\epsilon, H} \quad$ Since $u_{\epsilon}^{H, i}$ is defined as a minimizer on the convex set

$$
K=\left\{u \in W^{1, p}\left(Q_{H, i}\right) \mid<\nabla u>_{Q_{H, i}}=<\nabla u_{\epsilon}>_{Q_{H, i}}\right\},
$$

property (33) implies

$$
A_{i}^{\epsilon, H} \leq \frac{1}{c}\left|\int_{Q_{H, i}} W_{\epsilon}\left(x, \nabla u_{\epsilon}\right)-W_{\epsilon}\left(x, \nabla u_{\epsilon}^{H, i}\right)\right|
$$

In view of Lemmata 1 and 2 ,

$$
\lim _{\epsilon \rightarrow 0} \int_{Q_{H, i}} W_{\epsilon}\left(x, \nabla u_{\epsilon}\right)=\int_{Q_{H, i}} W_{h o m}\left(x, \nabla u_{h o m}\right) .
$$

Actually, as the locality of $\Gamma$-convergence and the liminf inequality imply

$$
\lim _{\epsilon \rightarrow 0} \int_{\Omega-Q_{H, i}} W_{\epsilon}\left(x, \nabla u_{\epsilon}\right) \leq \int_{\Omega-Q_{H, i}} W_{h o m}\left(x, \nabla u_{h o m}\right),
$$

$\lim _{\epsilon \rightarrow 0} \int_{Q_{H, i}} W_{\epsilon}\left(x, \nabla u_{\epsilon}\right)<\int_{Q_{H, i}} W_{h o m}\left(x, \nabla u_{h o m}\right)$ would contradict the convergence of the infima $\lim _{\epsilon \rightarrow 0} \int_{\Omega} W_{\epsilon}\left(x, \nabla u_{\epsilon}\right)=\int_{\Omega} W_{h o m}\left(x, \nabla u_{h o m}\right)$, which shows (44).

Let us prove that

$$
\lim _{\epsilon \rightarrow 0} \int_{Q_{H, i}} W_{\epsilon}\left(x, \nabla u_{\epsilon}^{H, i}\right)=\inf \left\{\int_{Q_{H, i}} W_{h o m}(x, \nabla u) \mid<\nabla u>_{Q_{H, i}}=<\nabla u_{h o m}>_{Q_{H, i}}\right\} .
$$

We have $<\nabla u_{\epsilon}>_{Q_{H, i}}=<\nabla u_{h o m}>_{Q_{H, i}}+\chi_{H, i}(\epsilon)$, where $\chi_{H, i}$ is a function that satisfies $\lim _{\epsilon \rightarrow 0}\left|\chi_{H, i}(\epsilon)\right|=0$ since $\nabla u_{\epsilon}$ weakly converges to $\nabla u_{\text {hom }}$ in $L^{p}(\Omega)$. 
In view of (37), we have

$$
\int_{Q_{H, i}} W_{\epsilon}\left(x, \nabla u_{h o m}^{H, i}+\chi_{H, i}(\epsilon)\right) \geq \int_{Q_{H, i}} W_{\epsilon}\left(x, \nabla u_{\epsilon}^{H, i}\right)
$$

since $<\nabla u_{\text {hom }}+\chi_{H, i}(\epsilon)>_{Q_{H, i}}=<\nabla u_{\epsilon}>_{Q_{H, i}}$.

Next we show:

$$
\begin{aligned}
& \left|\int_{Q_{H, i}} W_{\epsilon}\left(x, \nabla u_{h o m}^{H, i}+\chi_{H, i}(\epsilon)\right)-W_{\epsilon}\left(x, \nabla u_{h o m}^{H, i}\right)\right| \\
& \quad \leq \mathcal{C}\left|Q_{H, i}\right|\left|\chi_{H, i}(\epsilon)\right|\left(1+\left|<\nabla u_{h o m}>_{Q_{H, i}}\right|^{p-1}+\left|\chi_{H, i}(\epsilon)\right|^{p-1}\right) .
\end{aligned}
$$

To this end, let us start from (32):

$$
\begin{aligned}
& \left|W_{\epsilon}\left(x, \nabla u_{h o m}^{H, i}+\chi_{H, i}(\epsilon)\right)-W_{\epsilon}\left(x, \nabla u_{h o m}^{H, i}\right)\right| \\
& \quad \leq \mathcal{C}\left|\chi_{H, i}(\epsilon)\right|\left(1+\left|\nabla u_{h o m}^{H, i}+\chi_{H, i}(\epsilon)\right|^{p-1}+\left|\nabla u_{h o m}^{H, i}\right|^{p-1}\right) \\
& \quad \leq \mathcal{C}\left|\chi_{H, i}(\epsilon)\right|\left(1+\left|\chi_{H, i}(\epsilon)\right|^{p-1}+\left|\nabla u_{h o m}^{H, i}\right|^{p-1}\right),
\end{aligned}
$$

where $\mathcal{C}$ denotes various constants depending only on $c$ and $C$ in $H 3$. Integrating on $Q_{H, i}$ yields

$$
\begin{aligned}
\int_{Q_{H, i}} & \left|W_{\epsilon}\left(x, \nabla u_{h o m}^{H, i}+\chi_{H, i}(\epsilon)\right)-W_{\epsilon}\left(x, \nabla u_{h o m}^{H, i}\right)\right| \\
\leq & \int_{Q_{H, i}} \mathcal{C}\left|\chi_{H, i}(\epsilon)\right|\left(1+\left|\chi_{H, i}(\epsilon)\right|^{p-1}+\left|\nabla u_{h o m}^{H, i}\right|^{p-1}\right) \\
\leq & \mathcal{C}\left|\chi_{H, i}(\epsilon)\right|\left(\left|Q_{H, i}\right|\left(1+\left|\chi_{H, i}(\epsilon)\right|^{p-1}\right)+\int_{Q_{H, i}}\left|\nabla u_{h o m}^{H, i}\right|^{p-1}\right) .
\end{aligned}
$$

Successively using Hölder inequality, the minoration in H3, the definition (39) and the majoration in $H 3$, we have

$$
\begin{aligned}
\int_{Q_{H, i}}\left|\nabla u_{\text {hom }}^{H, i}\right|^{p-1} & \leq\left(\int_{Q_{H, i}}\left|\nabla u_{\text {hom }}^{H, i}\right|^{p}\right)^{\frac{p-1}{p}}\left(\int_{Q_{H, i}} 1\right)^{\frac{1}{p}} \\
& =\left|Q_{H, i}\right|^{\frac{1}{p}}\left(\int_{Q_{H, i}}\left|\nabla u_{\text {hom }}^{H, i}\right|^{p}\right)^{\frac{p-1}{p}} \\
& \leq \mathcal{C}\left|Q_{H, i}\right|^{\frac{1}{p}}\left(\int_{Q_{H, i}} W_{\epsilon}\left(x, \nabla u_{\text {hom }}^{H, i}(x)\right)\right)^{\frac{p-1}{p}} \\
& \leq \mathcal{C}\left|Q_{H, i}\right|^{\frac{1}{p}}\left(\int_{Q_{H, i}} W_{\epsilon}\left(x,<\nabla u_{\text {hom }}^{H, i}>_{Q_{H, i}}\right)\right)^{\frac{p-1}{p}} \\
& \leq \mathcal{C}\left|Q_{H, i}\right|^{\frac{1}{p}}\left(\left|Q_{H, i}\right|\left(1+\left|<\nabla u_{\text {hom }}^{H, i}>_{Q_{H, i}}\right|^{p}\right)\right)^{\frac{p-1}{p}} \\
& \leq \mathcal{C}\left|Q_{H, i}\right|^{\left(1+\left|<\nabla u_{\text {hom }}^{H, i}>Q_{H, i}\right|^{p-1}\right) .}
\end{aligned}
$$

$\mathrm{RR} \mathrm{n}^{\circ} 5791$ 
Inserting (49) into (48) shows (47).

Then we remark, in view of (39), that

$$
\left.\int_{Q_{H, i}} W_{\epsilon}\left(x, \nabla u_{h o m}^{H, i}\right) \leq \int_{Q_{H, i}} W_{\epsilon}\left(x, \nabla u_{\epsilon}^{H, i}-\chi_{H, i}(\epsilon)\right)\right)
$$

since $<\nabla u_{\epsilon}-\chi_{H, i}(\epsilon)>_{Q_{H, i}}=<\nabla u_{h o m}>_{Q_{H, i}}$.

Arguing as above for (47), we obtain:

$$
\begin{aligned}
& \left|\int_{Q_{H, i}} W_{\epsilon}\left(x, \nabla u_{\epsilon}^{H, i}-\chi_{H, i}(\epsilon)\right)-W_{\epsilon}\left(x, \nabla u_{\epsilon}^{H, i}\right)\right| \\
& \quad \leq C\left|Q_{H, i}\right|\left|\chi_{H, i}(\epsilon)\right|\left(1+\left|<\nabla u_{\epsilon}>_{Q_{H, i}}\right|^{p-1}+\left|\chi_{H, i}(\epsilon)\right|^{p-1}\right) .
\end{aligned}
$$

The combination of the four inequalities $(46),(47),(50)$ and (51) yields

$$
\begin{aligned}
& \left|\int_{Q_{H, i}} W_{\epsilon}\left(x, \nabla u_{h o m}^{H, i}\right)-W_{\epsilon}\left(x, \nabla u_{\epsilon}^{H, i}\right)\right| \\
& \leq \mathcal{C}\left|Q_{H, i}\right|\left|\chi_{H, i}(\epsilon)\right|\left(1+\left|<\nabla u_{h o m}>_{Q_{H, i}}\right|^{p-1}\right. \\
& \left.+\left|<\nabla u_{\epsilon}>_{Q_{H, i}}\right|^{p-1}+\left|\chi_{H, i}(\epsilon)\right|^{p-1}\right) .
\end{aligned}
$$

Letting $\epsilon$ go to zero in (52) proves inequality (45) since Lemmata 1 and 2 imply that

$$
\begin{aligned}
\lim _{\epsilon \rightarrow 0}\left(\inf \left\{\int_{Q_{H, i}} W_{\epsilon}(x, \nabla u) \mid<\nabla u>_{Q_{H, i}}=<\nabla u_{\text {hom }}>_{Q_{H, i}}\right\}\right) \\
=\inf \left\{\int_{Q_{H, i}} W_{h o m}(x, \nabla u) \mid<\nabla u>_{Q_{H, i}}=<\nabla u_{\text {hom }}>_{Q_{H, i}}\right\} .
\end{aligned}
$$

The combination of (43), (44) and (45) then shows

$$
0 \leq \limsup _{\epsilon \rightarrow 0} A_{i}^{\epsilon, H} \leq \mathcal{C}\left|\int_{Q_{H, i}} W_{\text {hom }}\left(x, \nabla u_{\text {hom }}\right)-W_{\text {hom }}\left(x, \nabla u_{\text {hom }}^{H, i}\right)\right| .
$$

We thus obtain

$$
\begin{aligned}
\limsup _{\epsilon \rightarrow 0} \sum_{i=1}^{I_{H}} A_{i}^{\epsilon, H} & \leq \mathcal{C} \sum_{i=1}^{I_{H}} \mid \int_{Q_{H, i}} W_{h o m}\left(x, \nabla u_{h o m}\right) \\
& -\inf \left\{\int_{Q_{H, i}} W_{h o m}(x, \nabla u) \mid<\nabla u>_{Q_{H, i}}=<\nabla u_{h o m}>_{Q_{H, i}}\right\} \mid .
\end{aligned}
$$

It remains to prove that the right hand side of (54) tends to 0 when $H$ goes to 0 .

Let $\bar{W}^{H}$ be the energy density defined by

$$
\bar{W}^{H}(x, \xi)=\sum_{i}^{I_{H}} \inf \left\{\frac{1}{\left|Q_{H, i}\right|} \int_{Q_{H, i}} W_{h o m}(x, \nabla u) \mid<\nabla u>=\xi\right\} 1_{Q_{H, i}}(x) .
$$

INRIA 
Arguing as in the proof of (27), for almost all $x \in \Omega$ and for all $\xi \in \mathbb{R}^{n}$, it can be shown that $\lim _{H \rightarrow 0} \bar{W}^{H}(x, \xi)=W_{\text {hom }}(x, \xi)$. Since every argument of the absolute values in the right hand side of (54) is positive, inequality (54) can be rewritten as

$$
\begin{array}{r}
\limsup _{\epsilon \rightarrow 0} \sum_{i=1}^{I_{H}} A_{i}^{\epsilon, H} \leq \mathcal{C}\left|\int_{\Omega} \bar{W}^{H}\left(x, M_{H}\left(\nabla u_{\text {hom }}\right)\right)-\bar{W}^{H}\left(x, \nabla u_{\text {hom }}\right)\right| \\
+\mathcal{C}\left|\int_{\Omega} \bar{W}^{H}\left(x, \nabla u_{\text {hom }}\right)-W_{\text {hom }}\left(x, \nabla u_{\text {hom }}\right)\right| .
\end{array}
$$

Let denote by $R h s_{1}^{H}$ and $R h s_{2}^{H}$ respectively the first and second terms of the righthand side of (55). Since $W_{\text {hom }}$ satisfies properties (2) and H3, we can argue as in the proof of (47) and obtain:

$$
\left|\bar{W}^{H}\left(x, \xi_{1}\right)-\bar{W}^{H}\left(x, \xi_{2}\right)\right| \leq \mathcal{C}\left|\xi_{1}-\xi_{2}\right|\left(1+\left|\xi_{1}\right|^{p-1}+\left|\xi_{2}\right|^{p-1}\right),
$$

which is independent of $H$. Thus, using Hölder inequality, $R h s_{1}^{H}$ is dominated by

$$
\mathcal{C}\left\|M_{H}\left(\nabla u_{h o m}\right)-\nabla u_{h o m}\right\|_{L^{p}(\Omega)}\left(1+\left\|M_{H}\left(\nabla u_{h o m}\right)\right\|_{L^{p}(\Omega)}^{p-1}+\left\|\nabla u_{h o m}\right\|_{L^{p}(\Omega)}^{p-1}\right)
$$

which converges to 0 as $H$ goes to 0 since $\lim _{H \rightarrow 0} M_{H}\left(\nabla u_{h o m}\right)=\nabla u_{h o m}$ in $L^{p}(\Omega)$.

Next, the dominated convergence theorem implies that $R h s_{2}^{H}$ also tends to 0 as $H$ goes to 0 , since the integrand pointwise converges to 0 and is dominated independently of $H$ using $H 3$.

This finally shows (41).

Limit of $\sum_{i=1}^{I_{H}} B_{i}^{\epsilon, H, \eta}$ Remark first that $<\nabla u_{\epsilon}>_{Q_{H, i}}=<\nabla u_{h o m}>_{Q_{H, i}}+\chi_{H, i}(\epsilon)$ and $<$ $\nabla v_{\eta, \epsilon}>_{Q_{H, i}}=<\nabla u_{h o m}>_{Q_{H, i}}+\phi_{H, i}(\epsilon, \eta)$ where $\chi_{H, i}$ and $\phi_{H, i}$ are functions satisfying $\lim _{\epsilon \rightarrow 0} \chi_{H, i}(\epsilon)=0$ and $\lim _{\eta \rightarrow 0} \lim _{\epsilon \rightarrow 0} \phi_{H, i}(\epsilon, \eta)=0$. Consequently, arguing as for (47), we obtain:

$$
\begin{aligned}
B_{i}^{\epsilon, H, \eta} \leq \mathcal{C}\left|Q_{H, i}\right|\left(\left|\chi_{H, i}(\epsilon)\right|+\left|\phi_{H, i}(\epsilon, \eta)\right|\right) \cdot\left(1+\left|<\nabla v_{\eta, \epsilon}>_{Q_{H, i}}\right|^{p-1}\right. \\
\left.+\left|<\nabla u_{\epsilon}>_{Q_{H, i}}\right|^{p-1}+\left|\chi_{H, i}(\epsilon)\right|^{p-1}+\left|\phi_{H, i}(\epsilon, \eta)\right|^{p-1}\right) .
\end{aligned}
$$

Letting $\epsilon$ go to 0 in (56) yields

$$
\begin{aligned}
\limsup _{\epsilon \rightarrow 0} B_{i}^{\epsilon, H, \eta} \leq \mathcal{C}\left|Q_{H, i}\right|\left|<\nabla v_{\eta, \text { hom }}>_{Q_{H, i}}-<\nabla u_{\text {hom }}>_{Q_{H, i}}\right| \\
\cdot\left(1+\left|<\nabla v_{\eta, h o m}>_{Q_{H, i}}\right|^{p-1}+\left|<\nabla u_{\text {hom }}>_{Q_{H, i}}\right|^{p-1}\right) .
\end{aligned}
$$

The summation of (57) for $i$ from 1 to $I_{H}$ exactly reads

$$
\begin{aligned}
& \limsup _{\epsilon \rightarrow 0} \sum_{i=1}^{I_{H}} B_{i}^{\epsilon, H, \eta} \leq \mathcal{C} \int_{\Omega}\left|M_{H}\left(\nabla v_{\eta, h o m}-\nabla u_{\text {hom }}\right)\right| \\
& \cdot\left(1+\left|M_{H}\left(\nabla v_{\eta, h o m}\right)\right|^{p-1}+\left|M_{H}\left(\nabla u_{\text {hom }}\right)\right|^{p-1}\right) .
\end{aligned}
$$

$\mathrm{RR} \mathrm{n}^{\circ} 5791$ 
Using Hölder inequality, we obtain

$$
\begin{aligned}
\limsup _{\epsilon \rightarrow 0} \sum_{i=1}^{I_{H}} B_{i}^{\epsilon, H, \eta} \leq & \mathcal{C}\left\|M_{H}\left(\nabla v_{\eta, h o m}-\nabla u_{\text {hom }}\right)\right\|_{L^{p}(\Omega)} \\
& \cdot\left(\int_{\Omega} 1+\left|M_{H}\left(\nabla v_{\eta, h o m}\right)\right|^{p}+\left|M_{H}\left(\nabla u_{h o m}\right)\right|^{p}\right)^{\frac{p-1}{p}} .
\end{aligned}
$$

Let us now prove that the right hand side of (59) converges to 0 uniformly in $H$ when $\eta$ goes to 0 . Using Hölder inequality, for every $w \in L^{p}(\Omega)$ and every $H>0$, we have

$$
\begin{aligned}
\int_{\Omega}\left|M_{H}(w)\right|^{p} & =\sum_{i} \mathcal{L}^{n}\left(Q_{H, i}\right)\left|\frac{1}{\mathcal{L}^{n}\left(Q_{H, i}\right)} \int_{Q_{H, i}} w\right|^{p} \\
& \leq \sum_{i} \mathcal{L}^{n}\left(Q_{H, i}\right)\left(\frac{1}{\mathcal{L}^{n}\left(Q_{H, i}\right)}\left(\int_{Q_{H, i}}|w|^{p}\right)^{1 / p}\left(\int_{Q_{H, i}} 1\right)^{(p-1) / p}\right)^{p} \\
& =\sum_{i} \int_{Q_{H, i}}|w|^{p} \\
& =\|w\|_{L^{p}(\Omega)}^{p} .
\end{aligned}
$$

This calculation shows that $\left(M_{H}\right)$ is an equicontinuous family of operators on $L^{p}(\Omega)$. By the application of Theorem $1, v_{\eta, h o m}$ converges strongly to $u_{\text {hom }}$ in $W^{1, p}(\Omega)$. This strong convergence and the equicontinuity of $M_{H}$ show that there exists $\mathcal{D}>0$ such that

$$
\left(\int_{\Omega} 1+\left|M_{H}\left(\nabla v_{\eta, h o m}\right)\right|^{p}+\left|M_{H}\left(\nabla u_{h o m}\right)\right|^{p}\right)^{\frac{p-1}{p}} \leq \mathcal{D}
$$

for every $\eta$ and $H$, and that

$$
\lim _{\eta \rightarrow 0}\left\|M_{H}\left(\nabla v_{\eta, h o m}-\nabla u_{\text {hom }}\right)\right\|_{L^{p}(\Omega)}=0
$$

uniformly in $H$, which proves (42).

The combination of (40), (41) and (42) concludes the proof of Theorem 2.

\section{Relation to some existing numerical approaches}

The analytical developments of the previous sections may serve as a theoretical framework for both the heterogeneous multiscale method (HMM) and the multiscale finite element method (MsFEM), when applied to the homogenization of elliptic operators. Indeed, as will be shown in Sections 3.1 and 3.2 respectively, the HMM and the MsFEM for elliptic 
equations can both basically be re-written as approximations by a quadrature rule of the energy functional

$$
\begin{aligned}
I_{\eta, \epsilon}(u)=\int_{\Omega} \inf \left\{<W_{\epsilon}(\cdot, \nabla v(\cdot))>_{B(x, \eta)} \mid\right. & \\
v & \left.\in W^{1, p}(B(x, \eta)),<\nabla v>_{B(x, \eta)}=\nabla u(x)\right\} d x
\end{aligned}
$$

introduced in Definition 3. This explains why the two methods give similar results as illustrated on some examples in [15].

A third existing methodology can be related to the theoretical framework developed in Sections 1 and 2, but it will not be detailed as much as the above two here. It is commonly used in mechanics, when dealing with linear composite materials. The method consists in introducing a small volume (namely a cube), called the representative volume element (RVE), and which represents the material at the macroscopic scale. The macroscopic stress-strain relation $\sigma(x)=A(x) \cdot \nabla u(x)$ of the material can then be approximated by the averaged response $<\sigma(y)>_{R V E}$ of the RVE under the homogeneous displacement $\nabla u(x) \cdot y$ of its boundary. In the present paper, the domain $B(x, \eta)$ plays the role of the RVE. As already mentionned, several boundary conditions can be associated to $W_{\eta, \epsilon}$ : at least periodic boundary conditions and Dirichlet boundary conditions. Other formulations can be developed using the Legendre transform of the energy and periodic or Dirichlet boundary conditions. In the limit $\epsilon$ and $\eta$ go to zero, all these energy densities converge to the same homogenized energy density. A huge body of literature in mechanics is devoted to the choice of $\eta$ and of the boundary conditions that best fit the behavior of the original material (see [17] for a review).

\section{$3.1 \quad \mathrm{HMM}$}

We first assume that $W_{\epsilon}$ is a strictly convex energy density and we describe the method within this context, for which minimizing the energy and solving the Euler-Lagrange equation are equivalent. The case of quasiconvex energies is the object of the end of this Section.

If we assume that $W_{\eta, \epsilon}$ is perfectly known, then the finite element approximation of

$$
\inf \left\{\int_{\Omega} W_{\eta, \epsilon}(x, \nabla u)-f u \mid u \in W^{1, p}(\Omega)+B C\right\}
$$

coincides with the method described in [7, Section 3] as an application of the HMM methodology to elliptic operators. Indeed, problem (60) is approximated at the discrete level by

$$
\inf \left\{\sum_{i=1}^{N_{m e s h}} \sum_{j=1}^{N_{G P}} q_{j}\left(W_{\eta, \epsilon}\left(x_{i j}, \nabla u_{H}\left(x_{i j}\right)\right)-f\left(x_{i j}\right) u_{H}\left(x_{i j}\right)\right), u_{H} \in V_{H}+B C\right\}
$$

where $N_{\text {mesh }}$ is the number of mesh elements, $N_{G P}$ is the number of Gauss points per element, $x_{i j}$ are the Gauss points, $q_{j}$ the weights and $V_{H}$ a finite element space. Then

$\mathrm{RR} \mathrm{n}^{\circ} 5791$ 
the computation of the FE minimizer of (61) only requires evaluations of derivatives of $W_{\eta, \epsilon}\left(x_{i j}, \xi\right)$ for particular $\xi$ at Gauss points $x_{i j}$. The first derivatives are needed for writing the Euler-Lagrange equation, the second derivatives if the latter is nonlinear and a Newtontype algorithm is used for the solution procedure. This is detailed in [14] where an explicit formula is given for periodic homogenization (the cell-problem is posed on $\left.(0,1)^{n}\right)$. The setting of [14] can easily be generalized replacing the periodic cell $(0,1)^{n}$ by $B(x, \eta)$.

The details of this general procedure may depend on the specific choice of $\eta$, of the shape of $B(x, \eta)$ (see Remark 1), and of the boundary conditions in the definition (6) of $W_{\eta, \epsilon}$ (see Remark 4). Many variants of the method follow.

The works [15] and [8] report some numerical experiments, where $B(x, \eta)$ is replaced by a cube and for which better numerical results are obtained using periodic boundary conditions instead of Dirichlet boundary conditions, even for the non-periodic problems tested.

Let us go back to the case for which $W_{\eta, \epsilon}$ is not analytically known but also numerically evaluated. We define a finite element approximation of $W_{\eta, \epsilon}(x, \xi)$ by

$$
W_{\eta, \epsilon}^{h}(x, \xi)=\inf \left\{<W_{\epsilon}\left(y, \xi+\nabla v_{h}\right)>_{B(x, \eta)} \mid v_{h} \in V_{h}\right\},
$$

where $V_{h}$ is a finite element subspace of $W^{1, p}(B(x, \eta))$ such that $\left\langle\nabla v_{h}>_{B(x, \eta)}=0\right.$ for all $v_{h} \in V_{h}$. On the theoretical side, the difference between the minimizer $u_{h o m}$ to the exact homogenized problem (5) and the finite element approximation $u_{\eta, H}^{h, \epsilon}$, defined as the minimizer of

$$
\inf \left\{\int_{\Omega} W_{\eta, \epsilon}^{h}\left(x, \nabla u_{H}\right)-f u_{H} \mid u_{H} \in V_{H}+B C\right\},
$$

may be decomposed into three components. Let denote by $u_{\eta}^{\epsilon}$ the minimizer of (60) and by $u_{\eta, H}^{\epsilon}$ the minimizer of

$$
\inf \left\{\int_{\Omega} W_{\eta, \epsilon}\left(x, \nabla u_{H}\right)-f u_{H} \mid u_{H} \in V_{H}+B C\right\} .
$$

Denoting by $\operatorname{err} 1(\eta, \epsilon)=\left\|u_{h o m}-u_{\eta}^{\epsilon}\right\|_{W^{1, p}(\Omega)}, \operatorname{err} 2(\eta, \epsilon, H)=\left\|u_{\eta}^{\epsilon}-u_{\eta, H}^{\epsilon}\right\|_{W^{1, p}(\Omega)}$ and by $\operatorname{err} 3(\eta, \epsilon, H, h)=\left\|u_{\eta, H}^{\epsilon}-u_{\eta, H}^{h, \epsilon}\right\|_{W^{1, p}(\Omega)}$, we then have by the triangle inequality

$$
\begin{aligned}
\left\|u_{h o m}-u_{\eta, H}^{h, \epsilon}\right\|_{W^{1, p}(\Omega)} \leq & \left\|u_{h o m}-u_{\eta}^{\epsilon}\right\|_{W^{1, p}(\Omega)}+\left\|u_{\eta}^{\epsilon}-u_{\eta, H}^{\epsilon}\right\|_{W^{1, p}(\Omega)} \\
& =\ln u_{\eta, H}^{\epsilon}-u_{\eta, H}^{h, \epsilon} \|_{W^{1, p}(\Omega)}
\end{aligned}
$$

The first component $\operatorname{err} 1$ has been studied in [7], [15] and [8] by Weinan E and collaborators in the framework of the HMM. The error analysis has been performed for elliptic operators linear in the gradient, that is operators of the form $u \mapsto a(x, u) \nabla u$. Under this 
assumption, the error, called $e(H M M)$, has been estimated in the periodic and stochastic cases.

The second component err 2 is the difference between the minimizer of (60) and its numerical FE approximation, the functional $W_{\eta, \epsilon}$ being considered as explicitly known. This analysis is more classical and can be performed without any assumption on the heterogeneities. This has also been addressed in [7], [15] and [8], where it is shown that err2 and $e(H M M)$ can interact nonlinearly.

The third component err 3 is the error due to the approximation of the energy density $W_{\eta, \epsilon}$ itself. In contrast to the difference between $W_{h o m}$ and $W_{\eta, \epsilon}$ which cannot be estimated in the general case, the error between $W_{\eta, \epsilon}$ and its numerical approximation $W_{\eta, \epsilon}^{h, \epsilon}$ can be estimated. This is also the case for the difference between the minimizers $u_{\eta, H}^{\epsilon}$ and $u_{\eta, H}^{h, \epsilon}$. In the nonlinear case, the contribution to err 3 of the difference between $W_{\eta, \epsilon}(x, \xi)$ and $W_{\eta, \epsilon}^{h}(x, \xi)$ can be magnified by the nonlinearity as shown in [14].

Except in the specific case of linear periodic homogenization (see [1]), a complete analysis of the global error $\left\|u_{\epsilon}-u_{\eta, H}^{h, \epsilon}\right\|_{L^{p}(\Omega)}$ seems out of reach.

Let us highlight the contribution of the present work in this setting. Theorem 1 shows that $\lim _{\eta \rightarrow 0} \lim _{\epsilon \rightarrow 0} \operatorname{err} 1(\eta, \epsilon)=0$ in the strictly convex case. It does not provide with quantitative estimates though. In addition, Theorem 2 shows that the reconstructed solution proposed in [8] is a numerical corrector. No further estimate can be derived without making more specific hypotheses on the dependence of $W_{\epsilon}$ upon the space variable.

Let us now make some remarks on the quasiconvex case. The numerical practice for such energy densities has been addressed in [14] in the setting of periodic homogenization. The method and results therein hold mutatis mutandis for non-periodic homogenization using the framework of Section 1.3. From a theoretical point of view, Theorem 4 is weaker than Theorem 1 since we have only proved the weak convergence of minimizers: $u_{\eta}^{h, \epsilon} \rightarrow u_{h o m}$ in $W^{1, p}(\Omega)$. However, there exist examples for which this convergence cannot be strong and that are linked to the possible loss of strict rank-one convexity by homogenization (see [13]).

\subsection{MsFEM}

Let $\left\{Q_{H, i}\right\}_{i}$ be a triangulation of $\Omega$ of size $H$. At each mesh element $Q_{H, i}$ we associate a point $x_{i} \in Q_{H, i}$. Given $u \in W^{1, p}(\Omega)$, we can approximate the integral $I_{\eta, \epsilon}(u)$ by

$$
\sum_{i}\left|Q_{H, i}\right| W_{\eta, \epsilon}\left(x_{i}, \nabla u\left(x_{i}\right)\right) .
$$

To obtain the MsFEM starting from (66), we have to make specific the value of $\eta$, the boundary conditions in (6) and the finite element space $V_{H}$. The specificity of the MsFEM 
with respect to the HMM is the link between $\eta$ and $H: \eta$ is taken to be $H$. The energy density used in the MsFEM is defined at $x_{i}$ by

$$
\inf \left\{\frac{1}{\left|Q_{H, i}\right|} \int_{Q_{H, i}} W_{\epsilon}(y, \nabla v) \mid v(y)=\xi \cdot y \text { on } \partial Q_{H, i}\right\}
$$

and denoted by $W_{H, \epsilon}^{M s F E M}\left(x_{i}, \xi\right)$ in the following. Taking $V_{H}$ as the space of $P 1$-finite elements on the triangulation $\left\{Q_{H, i}\right\}_{i}$, we obtain the discrete version of the MsFEM:

$$
\begin{aligned}
\inf \left\{\sum_{i=1}^{N_{\text {mesh }}}\left|Q_{H, i}\right| W_{H, \epsilon}^{M s F E M}\left(x_{i}, \nabla u_{H}\left(x_{i}\right)\right)\right. & \\
& \left.-\sum_{i=1}^{N_{\text {mesh }}} \sum_{j=1}^{N_{G P}} q_{j} f\left(x_{i j}\right) u_{H}\left(x_{i j}\right), u_{H} \in V_{H}+B C\right\},
\end{aligned}
$$

where the second term of the energy has been integrated by a quadrature rule associated to the triangulation. Problem (68) is to be compared to problem (61).

To prove the convergence of the MsFEM, Theorem 1 is not sufficient since both the finite element space $V_{H}$ and the numerical energy density $W_{H, \epsilon}^{M s F E M}$ depend on $H$. However an easy adaptation of the arguments of the proof of Theorem 1 allows to conclude. The rest of this section is devoted to such a proof in the general quasiconvex case, which has not been addressed by the authors of the method in their series of papers ([9],[10],[11] e.g.). Before we get to this, let us mention that we demonstrate convergence, but do not provide with any estimate of the error, even in the strictly convex case. This has been performed in [9] by Y. Efendiev and collaborators in a nonlinear setting for the specific case of periodic homogenization of monotone operators.

The following proof makes use of arguments that have been developed in other parts of the present work. They will only be sketched here. The new arguments are mainly linked to the convergence of the infima. We have also chosen to treat together the convergence in $\epsilon$ and $H$ in the presentation.

Adaptation of the proof of Theorem 3 The original nonlinear MsFEM (that is without the quadrature rule) can indeed be rewritten in the form:

$$
\inf \left\{\int_{\Omega} W_{H, \epsilon}^{M s F E M}\left(x, \nabla u_{H}\right)-f u_{H} \mid u_{H} \in V_{H}+B C\right\},
$$

if we extend the definition of $W_{H, \epsilon}^{M s F E M}$ on $\Omega$ by

$$
W_{H, \epsilon}^{M s F E M}(x, \xi)=\sum_{i} W_{H, \epsilon}^{M s F E M}\left(x_{i}, \xi\right) 1_{Q_{H, i}}(x),
$$

since $\nabla u_{H}$ is constant on each $Q_{H, i}$.

INRIA 
Let denote by $\left(P_{H}\right)$ an equi continuous family of projectors in $W^{1, p}(\Omega)$ associated to a family of P1-finite elements spaces $\left(V_{H},\|\cdot\|_{1, p}\right)$. In particular, there exists $C>0$ such that for all $u \in W^{1, p}(\Omega), \lim _{H \rightarrow 0}\left\|u-P_{H}(u)\right\|_{1, p}=0$ and $\left\|P_{H}(u)\right\|_{1, p} \leq C\|u\|_{1, p}$.

The same arguments as for the proof of Theorem 3 show that the family of energy functionals $I_{H, \epsilon}^{M S F E M}: u \mapsto \int_{\Omega} W_{H, \epsilon}^{M S F E M}\left(x, \nabla P_{H}(u)\right)-f P_{H}(u)$ is equi locally Lipschitz since $\left(P_{H}\right)$ is equi continuous on $W^{1, p}(\Omega)$. It converges pointwise on $W^{1, p}(\Omega)$ to the functional $\tilde{I}_{h o m}: u \mapsto \int_{\Omega} W_{h o m}(x, \nabla u)-f u$ as $\epsilon$ and $H$ go to 0 , by application of the dominated convergence theorem. Thus $I_{H, \epsilon}^{M s F E M} \Gamma\left(W^{1, p}\right)$-converges to $\tilde{I}_{h o m}$ by Lemma 8.

Next we prove the convergence of infima

$$
\begin{gathered}
\lim _{H \rightarrow 0} \lim _{\epsilon \rightarrow 0}\left(\inf \left\{\int_{\Omega} W_{H, \epsilon}^{M S F E M}\left(x, \nabla P_{H}(u)\right)-f P_{H}(u) \mid u \in W^{1, p}(\Omega)+B C\right\}\right) \\
=\inf \left\{\int_{\Omega} W_{h o m}(x, \nabla u)-f u \mid u \in W^{1, p}(\Omega)+B C\right\} .
\end{gathered}
$$

Denoting by $\tilde{V}_{H}=\left\{v \in W^{1, p}(\Omega) \mid v_{\mid \partial Q_{H, i}}\right.$ is linear for every $\left.i\right\},(67)$ implies

$$
\begin{aligned}
& \inf \left\{\int_{\Omega} W_{H, \epsilon}^{M s F E M}(x, \nabla u)-f u \mid u \in V_{H}+B C\right\} \\
& \quad=\inf \left\{\int_{\Omega} W_{\epsilon}(x, \nabla u)-f P_{H}(u) \mid u \in \tilde{V}_{H}+B C\right\} .
\end{aligned}
$$

Consequently,

$$
\begin{aligned}
& \inf \left\{\int_{\Omega} W_{H, \epsilon}^{M s F E M}(x, \nabla u)-f u \mid u \in V_{H}+B C\right\} \\
& \quad \geq \inf \left\{\int_{\Omega} W_{\epsilon}(x, \nabla u)-f P_{H}(u) \mid u \in W^{1, p}(\Omega)+B C\right\},
\end{aligned}
$$

which implies

$$
\begin{gathered}
\liminf _{H \rightarrow 0} \liminf _{\epsilon \rightarrow 0}\left(\inf \left\{\int_{\Omega} W_{H, \epsilon}^{M s F E M}(x, \nabla u)-f u \mid u \in V_{H}+B C\right\}\right) \\
\geq \inf \left\{\int_{\Omega} W_{h o m}(x, \nabla u)-f u \mid u \in W^{1, p}(\Omega)+B C\right\}
\end{gathered}
$$

since $u \mapsto P_{H}(u)$ is a continuous perturbation with respect to the topology of the $\Gamma\left(L^{p}\right)$ convergence (the liminf and the limsup inequalities still hold with the perturbation).

RR n $^{\circ} 5791$ 
Conversely, we have, for any minimizer $u_{\text {hom }}$ of the homogenized problem,

$$
\begin{aligned}
\limsup _{H \rightarrow 0} & \limsup _{\epsilon \rightarrow 0}\left(\inf \left\{\int_{\Omega} W_{H, \epsilon}^{M s F E M}\left(x, \nabla P_{H}(u)\right)-f P_{H}(u) \mid u \in W^{1, p}(\Omega)+B C\right\}\right) \\
\leq & \limsup _{H \rightarrow 0} \limsup _{\epsilon \rightarrow 0} \int_{\Omega} W_{H, \epsilon}^{M s F E M}\left(x, \nabla P_{H}\left(u_{h o m}\right)\right)-f P_{H}\left(u_{h o m}\right) \\
& =\inf \left\{\int_{\Omega} W_{h o m}(x, \nabla u)-f u \mid u \in W^{1, p}(\Omega)+B C\right\} .
\end{aligned}
$$

The combination of (71) and (72) shows (70).

From this point of the proof, we have to distinguish two cases: the strictly convex case and the general case.

In the strictly convex case, the same arguments as for the proof of the strong convergence of the equi isolated minimizers in Theorem 4 directly show the uniqueness of the minimizer for the homogenized energy and the strong convergence of the unique sequence of minimizers $u_{H, \epsilon} \in V_{H}+B C$ of

$$
\inf \left\{\int_{\Omega} W_{H, \epsilon}^{M s F E M}\left(x, \nabla P_{H}(u)\right)-f P_{H}(u) \mid u \in W^{1, p}(\Omega)+B C\right\}
$$

to $u_{\text {hom }}$ when $\epsilon$ and $H$ go to 0 .

For the general quasiconvex case, let us consider a sequence of minimizers $u_{H, \epsilon} \in V_{H}+B C$ of (73) and prove that it weakly converges, up to extraction, to some minimizer $u_{\text {hom }}$ of the homogenized problem. We skip the convergence in $\epsilon$ since the difficulty does not lye there. It remains to prove, with obvious notation, that the weak limit of $u_{H, h o m} \in V_{H}+B C$, still denoted by $u_{\text {hom }}$, is a minimizer of the homogenized problem. We already know that

$$
\lim _{H \rightarrow 0} I_{H, h o m}^{M s F E M}\left(u_{H, h o m}\right)=\inf \left\{\tilde{I}_{h o m}(u), u \in W^{1, p}(\Omega)+B C\right\}
$$

We have:

$$
I_{H, h o m}^{M s F E M}\left(u_{H, h o m}\right)-\tilde{I}\left(u_{h o m}\right)=I_{H, h o m}^{M s F E M}\left(u_{H, h o m}\right)-\tilde{I}\left(u_{H, h o m}\right)+\tilde{I}\left(u_{H, h o m}\right)-\tilde{I}\left(u_{h o m}\right) .
$$

Provided that

$$
\lim _{H \rightarrow 0}\left(I_{H, h o m}^{M s F E M}\left(u_{H, h o m}\right)-\tilde{I}\left(u_{H, h o m}\right)\right)=0,
$$

the weakly lower semicontinuity of $\tilde{I}_{h o m}$ implies that

$$
\liminf _{H \rightarrow 0} I_{H, h o m}^{M s F E M}\left(u_{H, h o m}\right) \geq \tilde{I}\left(u_{h o m}\right),
$$

which, combined with (74), shows that $u_{h o m}$ is a minimizer of the homogenized problem.

Actually, we do not exactly prove (75) for $u_{H, h o m}$ but for a $p$-equi-integrable sequence $v_{H, h o m}$ still weakly converging to $u_{\text {hom }}$ and given by Lemma 11 . Such a proof for a $p$-equiintegrable sequence is detailed twice in the Appendix (Sections 4.2 and 4.3) and not recalled 
here. We thus have $\liminf _{H \rightarrow 0} I_{H, h o m}^{M S F E M}\left(v_{H, h o m}\right) \geq \tilde{I}\left(u_{h o m}\right)$. In addition, outside a set $B_{H}$ such that $\lim _{H \rightarrow 0} \mathcal{L}^{n}\left(B_{H}\right)=0$ given by Lemma $11, \nabla v_{H, h o m}$ and $\nabla u_{H, h o m}$ coincide, thus

$$
\begin{aligned}
\int_{\Omega-B_{H}} W_{H, h o m}^{M s F E M}\left(x, \nabla v_{H, h o m}\right) & =\int_{\Omega-B_{H}} W_{H, h o m}^{M s F E M}\left(x, \nabla u_{H, h o m}\right) \\
& \leq \int_{\Omega} W_{H, h o m}^{M s F E M}\left(x, \nabla u_{H, h o m}\right) .
\end{aligned}
$$

The $p$-equi-integrability of $v_{H, h o m}$ and the growth condition (12) imply that

$$
\limsup _{H \rightarrow 0} \int_{\Omega-B_{H}} W_{H, h o m}^{M s F E M}\left(x, \nabla v_{H, h o m}\right)=\limsup _{H \rightarrow 0} \int_{\Omega} W_{H, h o m}^{M s F E M}\left(x, \nabla v_{H, h o m}\right) .
$$

Thus, $\limsup _{H \rightarrow 0} \int_{\Omega} W_{H, h o m}^{M s F E M}\left(x, \nabla v_{H, h o m}\right) \leq \lim _{H \rightarrow 0} \int_{\Omega} W_{H, h o m}^{M s F E M}\left(x, \nabla u_{H, h o m}\right)$ and

$$
\begin{aligned}
\tilde{I}_{\text {hom }}\left(u_{\text {hom }}\right) & \leq \liminf _{H \rightarrow 0} I_{H, h o m}^{M s F E M}\left(v_{H, h o m}\right) \\
& \leq \lim _{H \rightarrow 0} \int_{\Omega} W_{H, h o m}^{M s F E M}\left(x, \nabla u_{H, h o m}\right) \\
& =\inf \left\{\tilde{I}_{\text {hom }}(u), u \in W^{1, p}(\Omega)+B C\right\},
\end{aligned}
$$

which proves that $u_{\text {hom }}$ is a minimizer of the homogenized functional.

As for Theorem 4, we can also prove the strong convergence of the equi isolated minimizers. This concludes the extension of the proof of Thereom 3 to the MsFEM.

Concerning the numerical practice, as pointed out in [9], the multiscale finite elements can be computed on smaller domains than the macroscopic mesh elements and be extended by periodicity for instance. We refer to the bibliography for details. As far as general convergence results are concerned the MsFEM and the HMM for elliptic operators are equivalent in the present framework. We do not discuss here the practical implementation and the specificities of each method, for which we refer to the bibliography.

\section{Conclusion}

The setting of the present work is the minimization of an energy which depends on the gradient of a function. The prototypical example is hyperelasticity, for which the mechanical energy density of the material only depends on the gradient of deformation. More specifically we have considered an energy density that varies a lot spatially, which makes direct numerical simulations rather impossible to perform in practice. We have then introduced an alternative energy density, which is a kind of averaged or effective energy density. This energy density should have the advantage not to vary as much as the original one and is therefore easier to simulate numerically. These analytical developments have allowed us

$\mathrm{RR} \mathrm{n}^{\circ} 5791$ 
to recover some convergence results for the MsFEM in the convex case and to prove the corresponding results for the HMM. We have also extended the approach to nonlinear elasticity, by considering quasiconvex energy densities. Finally, we have proved that the usual reconstruction procedure to recover the fine scale features of the solution is indeed valid for general heterogeneities under classical monotonicity hypotheses.

\section{Acknowledgements}

The author would like to thank the IPAM, UCLA for its hospitality during the fall 2005, on the occasion of the long program "Bridging Time and Length Scales in Materials Science and Bio-Physics". 


\section{Contents}

1 Setting of the problem and statement of the main results 3

1.1 Homogenization of convex energy densities . . . . . . . . . . . . . . . . . . 4

1.2 Main results . . . . . . . . . . . . . . . . . . . . . 6

1.3 Extension to the quasiconvex case . . . . . . . . . . . 8

2 Proof of the main results $\quad 12$

2.1 The one-dimensional linear case . . . . . . . . . . . . . . . 12

2.2 Proof of Theorem 1 . . . . . . . . . . . . . . . . . 14

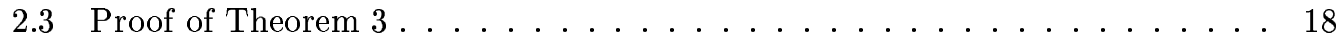

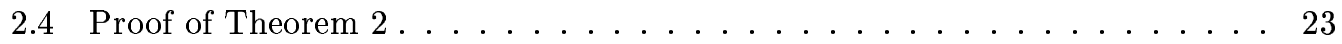

3 Relation to some existing numerical approaches 28

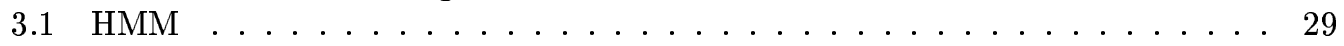

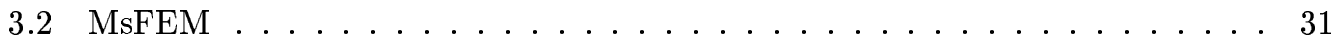

4 Appendix $\quad 39$

$4.1 \Gamma$-limit and boundary conditions . . . . . . . . . . . . . . . 39

4.2 Proof of Lemma $7[6$, Theorem 5.14] . . . . . . . . . . . . . . . . . . . . . . . . . . . . . . . . . . .

4.3 Proof of Lemma $12 \ldots \ldots \ldots \ldots 1$

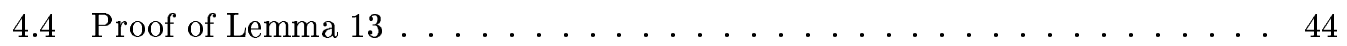




\section{References}

[1] A. ABDulLE, On a priori error analysis of fully discrete heterogeneous multiscale FEM, Multiscale Model. Simul., 4 (2005), pp. 447-459.

[2] E. Acerbi, And N. Fusco, Semicontinuity problems in the calculus of variations, Arch. Rat. Mech. Ana., 86 (1984), pp 125-145.

[3] M. BAÍA, AND I. FonseCA, The limit behavior of a family of variational multiscale problems, (http://www.math.cmu.edu/ nw0z/publications/05-CNA017/017abs/017abs.html), preprint (2005).

[4] A. Braides, and A. Defranceschi, Homogenization of Multiple Integrals, Oxford University Press, 1998.

[5] A. BRAIDES, Г-convergence for beginners, Oxford University Press, 2002.

[6] G. Dal Maso, An introduction to $\Gamma$-convergence, Birkhäuser, 1993.

[7] W. E, B. Engquist, X. Li, W. Ren, And E. VandenEIJNDEN, The Heterogeneous Multiscale Method: A Review, (http://www.math.princeton.edu/multiscale/review.pdf), preprint (2005).

[8] W. E, P.B. Ming, AND P.W. Zhang, Analysis of the heterogeneous multiscale method for elliptic homogenization problems, J. Amer. Math. Soc., 18 (2005), pp 121-156.

[9] Y.R. Efendiev, T.Y. Hou, And V. Ginting, Multiscale Finite Element Methods for Nonlinear Problems and their Applications, Com. Math. Sc., 2 (2004), No 4, pp 553-589.

[10] Y.R. Efendiev, and A. Pankov, Numerical Homogenization of Monotone Elliptic Operators, Multiscale Model. Simul., 2 (2003), No 1, pp 62-79.

[11] Y.R. Efendiev, And Pankov A., Numerical homogenization of nonlinear random parabolic operators, Multiscale Model. Simul., 2 (2004), No. 2, pp 237-268.

[12] I. Fonseca, S. Müller, And P. Pedregal, Analysis of concentration and oscillations effects generated by gradients, SIAM J. Math. Anal., 29 (1998), No. 3, pp 736-756.

[13] G. Geymonat G., S. Müller, and N. Triantafyllidis, Homogenization of Nonlinearly Elastic Materials, Microscopic Bifurcation and Macroscopic Loss of Rank-One Convexity, Arch. Rat. Mech. Anal., 122 (1993), pp 231-290.

[14] A. Gloria, A direct approach to numerical homogenization in finite elasticity, Networks and Heterogeneous Media, 1 (2006), No. 1, pp 109-141.

[15] P.B. Ming, AND X.Y. Yue, Numerical methods for multiscale elliptic problems, J. Comput. Physics, to appear. 
[16] M. OhlBerger, A posteriori error estimates for the heterogeneous multiscale finite element method for elliptic homogenization problems, Multiscale Model. Simul., 4 (2005), No. 1, pp 88-114.

[17] M. Ostoja-Starzewski, Material spatial randomness - from Statistical to Representative Volume Element, Prob. Eng. Mech., to appear, 2005.

[18] A. Pankov, G-convergence and Homogenization of Nonlinear Partial Differential Operators, Kluwer Academic Publishers, Dortrecht, The Netherlands, 1997.

[19] G. SAVARÉ, Regularity Results for Elliptic Equations in Lipschitz Domains, Journal of Functional Analysis, 152 (1998), pp 176-201.

\section{Appendix}

Besides a remark on the effect of boundary conditions on the $\Gamma$-limit, the present appendix contains, for consistency, the proof of Lemma 7 in the quasiconvex case, which is essentially the same of the one for the convex case in [6, Theorem 5.14], and the proof of the classical inequalities of Lemma 13. We also prove Lemma 12.

\section{1 $\Gamma$-limit and boundary conditions}

To prove that the $\Gamma$-limit on the spaces associated to (1), (2) and (3) is the same as the $\Gamma$ limit on $W^{1, p}(\Omega)$, it suffices to exhibit a recovery sequence which belongs to the variational space associated to the boundary conditions considered since Lemma 2 implies the liminf inequality is valid on the whole space $W^{1, p}(\Omega)$. We refer to [4, Proposition 11.7] where this issue is addressed for (1). The case (2) is a direct adaptation of the proof for (1) since as contructed in [4, Proposition 11.7], the recovery sequence can indeed by periodized if the limit $u$ is periodic. The case (3) can be dealt with as follows: given any $u \in\{v \in$ $\left.W^{1, p}(\Omega) \mid<\nabla v>_{\Omega}=\xi\right\}$, there exists a sequence $\left(u_{\epsilon}\right)$ such that $u_{\epsilon} \rightarrow u$ in $W^{1, p}(\Omega)$ and $\lim _{\epsilon \rightarrow 0} \int_{\Omega} W_{\epsilon}\left(x, \nabla u_{\epsilon}\right)=\int_{\Omega} W_{\text {hom }}(x, \nabla u)$ by the definition of $\Gamma$-convergence. Let then consider the following sequence: $\tilde{u}_{\epsilon}(x)=u_{\epsilon}(x)+\left(\xi-<\nabla u_{\epsilon}>_{\Omega}\right) \cdot x$. This sequence belongs to $\left\{v \in W^{1, p}(\Omega) \mid<\nabla v>_{\Omega}=\xi\right\}$ and weakly converges to $u$ in $W^{1, p}(\Omega)$. In addition, as a consequence of Lemma $6,\left\|\nabla u_{\epsilon}-\nabla \tilde{u}_{\epsilon}\right\|_{L^{p}(\Omega)}=\left|\xi-<\nabla u_{\epsilon}>_{\Omega}\right| \mathcal{L}^{n}(\Omega) \rightarrow 0$ implies that

$$
\lim _{\epsilon \rightarrow 0}\left|\int_{\Omega} W_{\tau}\left(x, \nabla u_{\epsilon}\right)-\int_{\Omega} W_{\tau}\left(x, \nabla \tilde{u}_{\epsilon}\right)\right|=0
$$

uniformly in $\tau$. Therefore,

$$
\lim _{\epsilon \rightarrow 0} \int_{\Omega} W_{\epsilon}\left(x, \nabla \tilde{u}_{\epsilon}\right)=\int_{\Omega} W_{h o m}(x, \nabla u),
$$

which proves that $\left(\tilde{u}_{\epsilon}\right)$ is a recovery sequence in $\left\{v \in W^{1, p}(\Omega) \mid<\nabla v>_{\Omega}=\xi\right\}$.

$\mathrm{RR} \mathrm{n}^{\circ} 5791$ 


\subsection{Proof of Lemma 7 [6, Theorem 5.14]}

This proof requires some basic properties of $\Gamma$-convergence, which can be read in the first chapter of [5]. We recall that Lemma 6 also holds for rank-one functions on $\mathbb{R}^{n \times d}$ with a different constant $K$ since any two matrices of $\mathbb{R}^{n \times d}$ are connected by at most $n d$ rank-one matrices.

By the dominated convergence theorem, $\lim _{\epsilon \rightarrow 0} \int_{\omega} \tilde{W}_{\epsilon}(x, \nabla u) d x=\int_{\omega} \tilde{W}(x, \nabla u) d x$ for every $u \in W^{1, p}(\omega)$. Thus, $\Gamma-\limsup _{\epsilon \rightarrow 0} \int_{\omega} \tilde{W}_{\epsilon}(x, \nabla u) d x \leq \int_{\omega} \tilde{W}(x, \nabla u) d x$. The conclusion is achieved if we prove that

$$
\int_{\omega} \tilde{W}(x, \nabla u) d x \leq \Gamma-\liminf _{\epsilon \rightarrow 0} \int_{\omega} \tilde{W}_{\epsilon}(x, \nabla u) d x
$$

Let us fix $u \in W^{1, p}(\Omega)$. By the absolute continuity of the integral for every $\theta>0$ there exists $\delta>0$ such that

$$
\int_{A}\left(|\nabla u|^{p}+1\right) d x<\theta
$$

for every mesurable subset $A$ of $\omega$ with $\mathcal{L}^{N}(A)<\delta$. Moreover, there exists $R>0$ such that $\mathcal{L}^{N}(\{|\nabla u| \geq R\})<\delta$.

Let $K=C\left((R+1)^{p}+1\right)$ and let $\xi_{1}, \ldots, \xi_{m}$ be points in the ball $B(0, R)$ such that

$$
B(0, R) \subseteq \cup_{i=0}^{m} B\left(\xi_{i}, \epsilon / K\right) .
$$

By the Egorov theorem, the sequences $\left(\tilde{W}_{\epsilon}\left(\cdot, \xi_{i}\right)\right)$ converge to $\tilde{W}\left(\cdot, \xi_{i}\right)$ quasi-uniformly on $\omega$. Therefore, there exist a measurable subset $A$ of $\omega$, with $\mathcal{L}^{N}(A)<\delta$, and a constant $k$ such that $\left|\tilde{W}_{\epsilon}\left(x, \xi_{i}\right)-\tilde{W}\left(x, \xi_{i}\right)\right| \leq \theta$ for every $x \in \omega \backslash A, i=1, \ldots, m$, and $\epsilon \leq 1 / k$. By (78) and by Lemma 6 , we obtain

$$
\left|\tilde{W}_{\epsilon}(x, \xi)-\tilde{W}(x, \xi)\right|<3 \theta
$$

for every $x \in \omega \backslash A$, for every $\xi \in B(0, R)$ and $\epsilon \leq 1 / k$.

Let $B=A \cup\{|\nabla u| \geq R\}$, let $g: \omega \times \mathbb{R}^{n} \rightarrow \mathbb{R}$ be the function defined by

$$
g(x, \xi)= \begin{cases}\tilde{W}(x, \xi), & \text { if } x \notin B, \\ 0, & \text { if } x \in B\end{cases}
$$

and let $G: W^{1, p}(\omega) \rightarrow \mathbb{R}$ be the corresponding integral functional, defined by

$$
G(u)=\int_{\omega} g(x, \nabla u) d x
$$

If $c=3 \mathcal{L}^{N}(\omega),(79)$ implies $\int_{\omega} \tilde{W}_{\epsilon}(x, \nabla u) d x+c \theta \geq G(u)$ for every $\epsilon \leq 1 / k$. As $G$ is lower semicontinuous for the weak topology of $W^{1, p}(\omega)$, we conclude that

$$
\left(\Gamma-\liminf _{\epsilon \rightarrow 0} \int_{\omega} \tilde{W}_{\epsilon}(x, \nabla u) d x\right)+c \theta \geq G(u) .
$$

INRIA 
Since $\int_{\omega} \tilde{W}(x, \nabla u) d x \leq G(u)+c_{1} \int_{B}\left(|\nabla u|^{p}+1\right) d x$, from (77) we get

$$
\int_{\omega} \tilde{W}(x, \nabla u) d x \leq G(u)+2 c_{1} \theta \leq\left(\Gamma-\liminf _{\epsilon \rightarrow 0} \int_{\omega} \tilde{W}_{\epsilon}(x, \nabla u) d x\right)+\left(c+2 c_{1}\right) \theta,
$$

so that (76) can be obtained by taking the limit as $\theta$ tends to 0 .

\subsection{Proof of Lemma 12}

We divide the proof in two steps. For almost every $x \in \mathcal{O}$ and for all $\xi \in \mathbb{R}^{n \times d}$, we first prove

$$
\limsup _{\epsilon \rightarrow 0} Q f_{\epsilon}(x, \xi) \leq Q f(x, \xi) .
$$

Then we prove the converse inequality

$$
\liminf _{\epsilon \rightarrow 0} Q f_{\epsilon}(x, \xi) \geq Q f(x, \xi) .
$$

Proof of inequality (80) For all $\epsilon>0$, almost every $x \in \mathcal{O}$ and for all $\xi \in \mathbb{R}^{n \times d}$, we have

$$
\begin{aligned}
Q f_{\epsilon}(x, \xi) & =\inf \left\{\int_{(0,1)^{n}} f_{\epsilon}(x, \xi+\nabla v(y)) d y \mid v \in W_{0}^{1, p}\left((0,1)^{n}, \mathbb{R}^{d}\right)\right\} \\
& \leq \int_{(0,1)^{n}} f_{\epsilon}(x, \xi+\nabla u(y)) d y
\end{aligned}
$$

for every $u \in W_{0}^{1, p}\left((0,1)^{n}, \mathbb{R}^{d}\right)$. Using the growth condition (12) and the dominated convergence theorem, we have

$$
\limsup _{\epsilon \rightarrow 0} Q f_{\epsilon}(x, \xi) \leq \int_{(0,1)^{n}} f(x, \xi+\nabla u(y)) d y .
$$

Since equation (83) holds for every $u \in W_{0}^{1, p}\left((0,1)^{n}, \mathbb{R}^{d}\right)$, we obtain

$$
\limsup _{\epsilon \rightarrow 0} Q f_{\epsilon}(x, \xi) \leq \inf \left\{\int_{(0,1)^{n}} f(x, \xi+\nabla v) \mid v \in W_{0}^{1, p}\left((0,1)^{n}, \mathbb{R}^{d}\right)\right\}=Q f(x, \xi) .
$$

Proof of inequality (81) Let us prove first that for all $\epsilon>0$, for all $\xi \in \mathbb{R}^{n \times d}$ and for all Lipschitz open subset $\omega$ of $\mathcal{O}$, there exists a sequence $\left\{\phi_{k}^{\epsilon}\right\}_{k} \in W^{1, p}\left(\omega, \mathbb{R}^{n}\right)$ such that

$$
\int_{\omega} Q f_{\epsilon}(x, \xi)=\lim _{k \rightarrow \infty} \int_{\omega} f_{\epsilon}\left(x, \nabla \phi_{k}^{\epsilon}\right)
$$

$\phi_{k}^{\epsilon} \rightarrow u_{\xi}$ in $W^{1, p}\left(\omega, \mathbb{R}^{n}\right)$, with $u_{\xi}(x)=\xi \cdot x$ on $\omega$, and satisfying the following properties:

$\mathrm{RR} \mathrm{n}^{\circ} 5791$ 
(a) there exists $\mathcal{C} \in \mathbb{R}$ such that for all $\epsilon>0$ and for all $k,\left\|\nabla \phi_{k}^{\epsilon}\right\|_{L^{p}(\omega)} \leq \mathcal{C}\left(1+|\xi|^{p}\right)$,

(b) for all $\epsilon$ and $k,\left\|\phi_{k}^{\epsilon}-u_{\xi}\right\|_{L^{p}(\omega)} \leq \frac{1}{k}$.

To this aim, we show that the sequence $\phi_{k}^{\epsilon}$ given by Lemma 10 satisfies (a) and (b) up to extraction. Property (b) is a direct consequence of the convergence of $\phi_{k}^{\epsilon}$ to $u_{\xi}$ in $L^{p}\left(\omega, \mathbb{R}^{n}\right)$. Using the growth condition (12), we have $\int_{\omega} f_{\epsilon}\left(x, \nabla \phi_{k}^{\epsilon}\right) \geq c\left\|\nabla \phi_{k}^{\epsilon}\right\|_{L^{p}(\omega)}$ and $\int_{\omega} Q f_{\epsilon}(x, \xi) \leq \int_{\omega} f_{\epsilon}(x, \xi) \leq C\left(1+|\xi|^{p}\right) \mathcal{L}^{n}(\omega)$, where the constants $c$ and $C$ do not depend on $\epsilon$, which implies property (a) for some $\mathcal{C} \in \mathbb{R}$.

As $Q f_{\epsilon}$ satisfies (12) uniformly in $\epsilon$ (the lower bound in (12) is a convex function lower or equal to $f_{\epsilon}$ thus it is also lower or equal to its quasiconvex envelop), there exists a subsequence $\epsilon_{j}$ and $L \in \mathbb{R}$ such that $\lim _{j \rightarrow \infty} \int_{\omega} Q f_{\epsilon_{j}}(x, \xi)=L$. Therefore, $\lim _{j \rightarrow \infty} \lim _{k \rightarrow \infty} \int_{\omega} f_{\epsilon_{j}}\left(x, \nabla \phi_{k}^{\epsilon_{j}}\right)$ exists and a diagonal extraction argument shows there exists an extraction function $\pi$ such that $\lim _{k \rightarrow \infty} \pi(k)=+\infty$ and

$$
\lim _{k \rightarrow \infty} \int_{\omega} f_{\epsilon_{\pi(k)}}\left(x, \nabla \phi_{k}^{\epsilon_{\pi(k)}}\right)=L
$$

As the sequence $\phi_{k}^{\epsilon_{\pi(k)}}$ satisfies properties (a) and (b), it is bounded in $W^{1, p}\left(\omega, \mathbb{R}^{n}\right)$ and it satisfies $\lim _{k \rightarrow \infty} \phi_{k}^{\epsilon_{\pi(k)}}=u_{\xi}$ in $L^{p}\left(\omega, \mathbb{R}^{n}\right)$. Up to a further extraction, we may suppose that $\phi_{k}^{\epsilon_{\pi(k)}}$ converges weakly to $u_{\xi}$ in $W^{1, p}\left(\omega, \mathbb{R}^{n}\right)$ by the uniqueness of the limit in the sense of distributions.

Applying now Lemma 11, we obtain the existence of a sequence $\chi_{k} \in W^{1, p}\left(\omega, \mathbb{R}^{n}\right)$ such that $\nabla \chi_{k}$ is $p$-equi-integrable, $\chi_{k}$ weakly converges to $u_{\xi}$ in $W^{1, p}\left(\omega, \mathbb{R}^{n}\right)$ and satisfies

$$
\lim _{k \rightarrow \infty} \mathcal{L}^{N}\left(\left\{x \in \omega: \nabla \chi_{k}(x) \neq \nabla \phi_{k}^{\epsilon_{\pi(k)}}(x)\right\}\right)=0,
$$

up to a further extraction.

Since $f_{\epsilon} \geq 0$,

$$
\begin{aligned}
\lim _{k \rightarrow \infty} \int_{\omega} f_{\epsilon_{\pi(k)}}\left(x, \nabla \phi_{k}^{\epsilon_{\pi(k)}}\right) & \geq \limsup _{k \rightarrow \infty} \int_{\left\{x \in \omega: \nabla \chi_{k}(x)=\nabla \phi_{k}^{\left.\epsilon_{\pi(k)}(x)\right\}}\right.} f_{\epsilon_{\pi(k)}}\left(x, \nabla \chi_{k}\right) \\
& \geq \limsup _{k \rightarrow \infty} \int_{\omega} f_{\epsilon_{\pi(k)}}\left(x, \nabla \chi_{k}\right),
\end{aligned}
$$

the last inequality being a consequence of the $p$-equi-integrability of $\nabla \chi_{k}$, the growth condition (12) and equation (84).

Next we prove that

$$
\lim _{\epsilon \rightarrow 0} \int_{\omega} f_{\epsilon}\left(x, \nabla \chi_{k}\right)=\int_{\omega} f\left(x, \nabla \chi_{k}\right)
$$


uniformly in $k$. From (86), we will deduce

$$
L \geq \limsup _{k \rightarrow \infty} \int_{\omega} f_{\epsilon_{\pi(k)}}\left(x, \nabla \chi_{k}\right) \geq \liminf _{k \rightarrow \infty} \int_{\omega} f\left(x, \nabla \chi_{k}\right) \geq \int_{\omega} Q f(x, \xi),
$$

since $v \mapsto \int_{\omega} Q f(x, \nabla v)$ is the lower-semicontinuous envelop of $v \mapsto \int_{\omega} f(x, \nabla v)$ for the weak topology of $W^{1, p}\left(\omega, \mathbb{R}^{n}\right)$ and $\chi_{k} \rightarrow u_{\xi}$ in $W^{1, p}\left(\omega, \mathbb{R}^{n}\right)$.

The proof of (86) follows the first part of the proof of Lemma 7, it relies on the $p$-equiintegrability of $\nabla \chi_{k}$ and on the fact that $\left(f_{\epsilon}\right)$ are equi-Lipschitz functions.

The $p$-equi-integrability of $\nabla \chi_{k}$ implies that for all $\theta>0$ there exists $\delta>0$ such that for all $k>0, \int_{A}\left(\left|\nabla \chi_{k}\right|^{p}+1\right) d x<\theta$ for every measurable subset $B$ of $\omega$ with $\mathcal{L}^{N}(B)<\delta$. Moreover, as $\nabla \chi_{k}$ is a bounded sequence in $L^{p}(\omega)$, there also exists $R>0$ such that for all $k>0, \mathcal{L}^{N}\left\{x \in \omega:\left|\nabla \chi_{k}(x) \geq R\right|\right\}<\delta$.

By assumption, there exists $K>0$ such that for all $\epsilon>0, f_{\epsilon}$ and $f$ are $K$-Lipschitz on $B(0, R)$ for almost every $x \in \omega$. Let $\xi_{1}, \ldots, \xi_{m}$ be points in the ball $B(0, R)$ such that

$$
B(0, R) \subseteq \cup_{i=0}^{m} B\left(\xi_{i}, \epsilon / K\right) .
$$

By the Egorov theorem, the sequences $f_{\epsilon}\left(\cdot, \xi_{i}\right)$ converge to $f\left(\cdot, \xi_{i}\right)$ quasi-uniformly on $\omega$. Therefore, there exist a measurable set $A$ of $\omega$, with $\mathcal{L}^{N}(A)<\delta$, and an integer $\kappa$ such that $\left|f_{\epsilon}\left(x, \xi_{i}\right)-f\left(x, \xi_{i}\right)\right| \leq \theta$ for every $x \in \omega \backslash A, i=1, \ldots, m$, and $\epsilon \leq 1 / \kappa$. By (88) and by the $K$-Lipschitz properties of $f_{\epsilon}$ and $f$, we obtain

$$
\left|f_{\epsilon}(x, \xi)-f(x, \xi)\right|<3 \theta
$$

for every $x \in \omega \backslash A$, for every $\xi \in B(0, R)$ and $\epsilon \leq 1 / \kappa$.

The dominated convergence theorem, inequality (89), the majoration in (12) with the constant $C$ and the definition of $A$ and $R$ imply that for all $\theta>0$, there exists $\kappa \in \mathbb{N}$ such that for all $\epsilon<1 / \kappa$ and $k>0$,

$$
\int_{\omega}\left|f_{\epsilon}\left(x, \nabla \chi_{k}\right)-f\left(x, \nabla \chi_{k}\right)\right| \leq\left(3 \mathcal{L}^{N}(\omega)+2 C\right) \theta,
$$

which proves (86).

Finally, as (87) holds for any converging subsequence of $\int_{\omega} Q f_{\epsilon_{j}}(x, \xi)$ and for any Lipschitz open subset $\omega$ of $\mathcal{O}$, we obtain (81). This concludes the proof of Lemma 12.

$\mathrm{RR} \mathrm{n}^{\circ} 5791$ 


\subsection{Proof of Lemma 13}

This proof is based on elementary calculus.

$$
\begin{aligned}
\left|W\left(x, \xi_{1}\right)-W\left(x, \xi_{2}\right)\right| & =\left|\int_{0}^{1} a\left(x, \xi_{1}+t\left(\xi_{2}-\xi_{1}\right)\right) \cdot\left(\xi_{2}-\xi_{1}\right) \mathrm{d} t\right| \\
& \leq C\left|\xi_{2}-\xi_{1}\right|\left(1+\left|\xi_{1}\right|^{p-1}+\left|\xi_{2}-\xi_{1}\right|^{p-1}\right)
\end{aligned}
$$

for all $\alpha \geq 0$ in (2).

We now prove inequality (33). Let $u$ minimize inf $\left\{\int_{\Omega} W(x, \nabla v) \mid v \in K\right\}$. Since for every $v \in K, \int_{\Omega} a(x, \nabla u) \cdot(\nabla v-\nabla u) \geq 0$ holds, (33) is a consequence of the following inequality:

$$
\int_{\Omega} W(x, \nabla u)-\int_{\Omega} W(x, \nabla v)-\int_{\Omega} a(x, \nabla u) \cdot(\nabla v-\nabla u) \geq c\|\nabla u-\nabla v\|_{L^{p}(\Omega)}^{p} .
$$

For every $v \in K$, let us introduce a function

$$
g:[0,1] \rightarrow \mathbb{R}, t \mapsto g(t)=\int_{\Omega} a(x, \nabla u+t(\nabla v-\nabla u)) .
$$

This function is real, differential and convex. As

$$
g^{\prime}(t)=\int_{\Omega} a(x, \nabla u+t(\nabla v-\nabla u)) \cdot(\nabla v-\nabla u),
$$

we have

$$
\begin{aligned}
& \int_{\Omega} W(x, \nabla u)-\int_{\Omega} W(x, \nabla v)-\int_{\Omega} a(x, \nabla u) \cdot(\nabla v-\nabla u) \\
= & g(1)-g(0)-g^{\prime}(0) \\
= & \int_{0}^{1}\left(g^{\prime}(t)-g^{\prime}(0)\right) \mathrm{d} t \\
= & \int_{0}^{1} \int_{\Omega}(a(x, \nabla u+t(\nabla v-\nabla u))-a(x, \nabla u)) \cdot t(\nabla v-\nabla u) \frac{\mathrm{d} t}{t} \\
\geq & c \int_{0}^{1}\|\nabla v-\nabla u\|_{L^{p}(\Omega)}^{p} t^{p-1} \mathrm{~d} t \\
\geq & c\|\nabla v-\nabla u\|_{L^{p}(\Omega)}^{p},
\end{aligned}
$$

since property (3) also implies $\left(a\left(x, \xi_{1}\right)-a\left(x, \xi_{2}\right)\right) \cdot\left(\xi_{1}-\xi_{2}\right) \geq c\left|\xi_{1}-\xi_{2}\right|^{p}$ for $\beta \leq p$. This proves (91) and consequently (33).

INRIA 


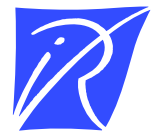

Unité de recherche INRIA Rocquencourt

Domaine de Voluceau - Rocquencourt - BP 105 - 78153 Le Chesnay Cedex (France)

Unité de recherche INRIA Futurs : Parc Club Orsay Université - ZAC des Vignes

4, rue Jacques Monod - 91893 ORSAY Cedex (France)

Unité de recherche INRIA Lorraine : LORIA, Technopôle de Nancy-Brabois - Campus scientifique

615, rue du Jardin Botanique - BP 101 - 54602 Villers-lès-Nancy Cedex (France)

Unité de recherche INRIA Rennes : IRISA, Campus universitaire de Beaulieu - 35042 Rennes Cedex (France)

Unité de recherche INRIA Rhône-Alpes : 655, avenue de l'Europe - 38334 Montbonnot Saint-Ismier (France)

Unité de recherche INRIA Sophia Antipolis : 2004, route des Lucioles - BP 93 - 06902 Sophia Antipolis Cedex (France)

INRIA - Domaine de Voluceau - Rocquencourt, BP 105 - 78153 Le Chesnay Cedex (France)

http://www.inria.fr

ISSN 0249-6399 